\title{
Fuzzy Soft Connected Sets in Fuzzy Soft Topological Spaces I
}

\author{
A. Kandil $^{1}$, O. A. E. Tantawy ${ }^{2}$, S. A. El-Sheikh ${ }^{3}$ and Sawsan S. S. El-Sayed ${ }^{4}$ \\ ${ }^{1}$ Mathematics Department, Faculty of Science, Helwan University, Helwan, Egypt \\ dr.ali_kandil@yahoo.com \\ 2 Mathematics Department, Faculty of Science, Zagazig University, Zagazig, Egypt \\ drosamat@yahoo.com \\ 3 \\ Mathematics Department, Faculty of Education, Ain Shams University, Cairo, Egypt \\ elsheikh33@hotmail.com \\ ${ }^{4}$ Mathematics Department, Faculty of Education, Ain Shams University, Cairo, Egypt \\ sawsan_809@yahoo.com
}

\begin{abstract}
In this paper we introduce some types of fuzzy soft separated sets and study some of thier preperties. Next, the notion of connectedness in fuzzy topological spaces due to Ming and Ming, Zheng etc., extended to fuzzy soft topological spaces. The relationship between these types of connectedness in fuzzy soft topological spaces is investigated with the help of number of counter examples.
\end{abstract}

Keywords: Fuzzy soft sets; fuzzy soft topological space; fuzzy soft separated sets; fuzzy soft Q-separated sets; fuzzy soft weakly separated sets; fuzzy soft strongly separated sets; fuzzy soft connected sets.

\section{INTRODUCTION}

After Zadeh [26] introduced the notion of a fuzzy set in 1965, Chang [4] used that concept to define fuzzy topology. In 1999, Molodtsov [15] introduced the concept of soft set theory which is a completely new approach for modeling uncertainty. In [15], Molodtsov established the fundamental results of this new theory and successfully applied the soft set theory into several directions, such as smoothness of functions, operations research, Riemann integration, game theory, theory of probability and so on. Maji et al. [13] defined and studied several basic notions of soft set theory in 2003. Shabir and Naz [21] introduced the concept of soft topological space.

Maji et al. [12] initiated the study involving both fuzzy sets and soft sets. In this paper, the notion of fuzzy soft sets was introduced as a fuzzy generalizations of soft sets and some basic properties of fuzzy soft sets are discussed in detail. Maji et al. combined fuzzy sets and soft sets and introduced the concept of fuzzy soft sets. To continue the investigation on fuzzy soft sets, Ahmad and Kharal [1] presented some more properties of fuzzy soft sets and introduced the notion of a mapping on fuzzy soft sets. In 2011, Tanay et al. [22] gave the topological structure of fuzzy soft sets.

The notion of connectedness in fuzzy topological spaces has been studied by Ming and Ming [14], Lowen [10], Zheng Chong You [28], Fatteh and Bassan [7], Zhao [27], Saha [20], Ajmal and Kohli [2], and Chaudhuri and Das [6]. In soft setting, the notion of soft connectedness introduced by many authers such as Peyghan et al. [18], Yüksel et al. [25]. In 2013, Bayramov et al. [4] studied the soft path connectedness on soft topological spaces. In 2014, Munir et al. [16] studied some properties of soft connected spaces and soft locally connected spaces. In 2015, Hussain [8] wrote a paper entitled a note on soft connectedness. In fuzzy soft setting, connectedness has been introduced by Mahanta [11] and Karatas et al. [9].

In this paper, we extend the notion of connectedness of fuzzy topological space to fuzzy soft topological space. In Section 3 , we introduce the different notions of fuzzy soft separated sets and study the relationship between them. Section 4 is devoted to introduce the different notions of connectedness in fuzzy soft topological space and study the implications that exist between them. Also, we study the characterization of connectedness in fuzzy soft setting.

\section{PRELIMINARIES}

Throughout this paper $X$ denotes initial universe, $E$ denotes the set of all possible parameters which are attributes,characteristic or properties of the objects in $X$, and the set of all subsets of $X$ will be denoted by $P(X)$. In this section, we present the basic definitions and results of soft set theory which will be needed in the sequel.

Definition 2.1. [5] A fuzzy set $A$ of a non-empty set $X$ is characterized by a membership function $\mu_{A}: X \rightarrow[0,1]=I$ whose value $\mu_{A}(x)$ represents the "degree of membership" of $x$ in $A$ for $x \in X$. Let $I^{X}$ denotes the family of all fuzzy sets on $X$.

Defintion 2.2. [15] Let $A$ be a non-empty subset of $E$. A pair $(F, A)$ denoted by $F_{A}$ is called a soft set over $X$, where $F$ is a mapping given by $F: A \rightarrow P(X)$. In other words, a soft set over $X$ is a parametrized family of subsets of 
the universe $X$. For a particular $e \in A, F(e)$ may be considered the set of $e$-approximate elements of the soft set $(F, A)$ and if $e \notin A$, then $F(e)=\phi$. i.e., $F=\{F(e): e \in A \subseteq E, F: A \rightarrow P(X)\}$.

Aktaş and Çağman [3] showed that every fuzzy set may be considered as a soft set. That is, fuzzy sets are a special class of soft sets.

Definition 2.3. [12] Let $A \subseteq E$. A pair $(f, A)$, denoted by $f_{A}$, is called fuzzy soft set over $X$, where $f$ is a mapping given by $f: A \rightarrow I^{X}$ defined by $f_{A}(e)=\mu_{f_{A}}^{e} ;$ where $\mu_{f_{A}}^{e}=\overline{0}$ if $e \notin A$, and $\mu_{f_{A}}^{e} \neq \overline{0}$ if $e \in A_{\text {, where }}$ $\overline{0}(x)=0 \forall x \in X$. The family of all these fuzzy soft sets over $X$ denoted by $\operatorname{FSS}(X)_{E}$.

Definition 2.4. $[12,17,19,22,23,24]$ The complement of a fuzzy soft set $(f, A)$, denoted by $(f, A)^{c}$, and defined by $(f, A)^{c}=\left(f^{c}, A\right), f_{A}^{c}: A \rightarrow I^{X}$ is a mapping given by $\mu_{f_{A}^{c}}^{e}=1-\mu_{f_{A}}^{e} \forall e \in A$. Clearly, $\left(f_{A}^{c}\right)^{c}=f_{A^{*}}$

Definition 2.5. $[12,17,19,22,24]$ A fuzzy soft set $f_{E}$ over $X$ is said to be a null- fuzzy soft set, denoted by $\tilde{0}_{E}$, if for all $e \in E, f_{E}(e)=\overline{0}$.

Definition 2.6. $[12,17,19,22,24]$ A fuzzy soft set $f_{E}$ over $X$ is said to be an absolute fuzzy soft set, denoted by $\tilde{1}_{E}$, if $f_{A}(e)=\overline{1}^{\forall e} \in E$. Clearly, we have $\left(\tilde{0}_{E}\right)^{c}=\tilde{1}_{E}$ and $\left(\tilde{1}_{E}\right)^{c}=\tilde{0}_{E^{*}}$

Definition 2.7. $[12,17,19,22,23,24]$ Let $f_{A}$ and $g_{B} \in \operatorname{FSS}(X)_{E}$. Then $f_{A}$ is fuzzy soft subset of $g_{B}$, denoted by $f_{A} \subseteq g_{B}$, if $A \subseteq B$ and $\mu_{f_{A}}^{e}(x) \leq \mu_{g_{B}}^{e}(x) \forall x \in X, \forall e \in E$. Also, $g_{B}$ is called fuzzy soft superset of $f_{A}$ denoted by $g_{B} \supseteq f_{A}$. If $f_{A}$ is not fuzzy soft subset of $g_{B}$, we written as $f_{A} \not \subset g_{B}$.

Definition.2.8. $[12,17,19,22,23,24]$ Two fuzzy soft sets $f_{A}$ and $g_{B}$ on $X$ are called equal if $f_{A} \subseteq g_{B}$ and $g_{B}$ $\subseteq f_{A}$.

Definition 2.9. $[12,17,19,22,24]$ The union of two fuzzy soft sets $f_{A}$ and $g_{B}$ over the common universe $X$, denoted by $f_{A} \cup g_{B}$, is also a fuzzy soft set $h_{C}$, where $C=A \cup B$ and for all $e \in C$, $h_{C}(e)=\mu_{h_{C}}^{e}=\mu_{f_{A}}^{e} \vee \mu_{g_{B}}^{e}$

Definition 2.10. $[12,17,19,22,24]$ The intersection of two fuzzy soft sets $f_{A}$ and $g_{B}$ over the common universe $X$, denoted by $f_{A} \widetilde{\cap} g_{B}$, is also a fuzzy soft set $h_{C}$, where $C=A \cap B$ and for all $e \in C, h_{C}(e)=\mu_{h_{C}}^{e}=\mu_{f_{A}}^{e} \wedge \mu_{g_{B}}^{e}$.

Definition 2.12. [22] Let $F S S(X)_{E}$ be a collection of fuzzy soft sets over a universe $X$ with a fixed set of parameters $E$. Then $\tau \subseteq F S S(X)_{E}$ is called fuzzy soft topology on $X$ if:

(1) $\tilde{0}_{E}, \tilde{1}_{E} \in \tau$, where $\tilde{0}_{E}(e)=\overline{0}$ and $\tilde{1}_{E}(e)=\overline{1} \forall e \in E$,

(2) The union of any members of $\tau$ belongs to $\tau$.

(3) The intersection of any two members of $\tau$ belongs to $\tau$.

The triplet $(X, \tau, E)$ is called fuzzy soft topological space over $X$. Also, each member of $\tau$ is called fuzzy soft open set in $(X, \tau, E)$.

Examples 2.1. The following are fuzzy soft topology on $X$ :

(1) $\tau_{0}=\left\{\tilde{0}_{E}, \tilde{1}_{E}\right\}$ is called fuzzy soft indiscrete topology on $X$

(2) $\tau_{D}=\operatorname{FSS}(X)_{E}$ is called fuzzy soft discrete topology on $X$.

Note that, the intersection of any family of fuzzy soft topologies on $X$ is also a fuzzy soft topology on $X$.

Definition 2.13. [22] Let $(X, \tau, E)$ be a fuzzy soft topological space. A fuzzy soft set $f_{A}$ over $X$ is said to be fuzzy soft closed set in $X$, if its relative complement $f_{A}^{c}$ is fuzzy soft open set. The collection of all fuzzy soft closed sets will be denoted by $\tau^{c}$. 
Definition 2.14. $[17,19] \operatorname{Let}(X, \tau, E)$ be a fuzzy soft topological space and $f_{A} \in F S S(X)_{E}$. The fuzzy soft closure of $f_{A}$, denoted by $\operatorname{Fcl}\left(f_{A}\right)$, is the intersection of all fuzzy soft closed supersets of $f_{A}$. i.e., $\operatorname{Fcl}\left(f_{A}\right)=\widetilde{\cap}\left\{h_{C} ; h_{C} \in \tau^{c}, f_{A} \subseteq h_{C}\right\}$. Clearly, $\operatorname{Fcl}\left(f_{A}\right)$ is the smallest fuzzy soft closed set over $X$ which contains $f_{A}$, and $\operatorname{Fcl}\left(f_{A}\right)$ is fuzzy soft closed set.

Definition 2.15. [19, 24] The fuzzy soft set $f_{A} \in \operatorname{FSS}(X)_{E}$ is called fuzzy soft point if there exist $x \in X$ and $e \in E$ such that $\mu_{f_{A}}^{e}(x)=\alpha ;(0 \leq \alpha \leq 1)$ and $\mu_{f_{A}}^{e}(y)=0 \forall y \in X-\{x\}$ and this fuzzy soft point is denoted by $x_{\alpha}^{e}$ or $f_{\theta}$. The class of all fuzzy soft points of $X$, denoted by $\operatorname{FSP}(X)_{E}$.

Definition 2.16. [11] The fuzzy soft point $x_{\alpha}^{e}$ is said to be belonging to the fuzzy soft set $f_{A}$, denoted by $x_{\alpha}^{e} \widetilde{\in} f_{A}$, if for the element $e \in A, \alpha \leq \mu_{f_{A}}^{e}(x)$. If $x_{\alpha}^{\theta}$ is not belong to $f_{A}$, we write $x_{\alpha}^{e} \widetilde{\notin} f_{A}$ and implies that $\alpha>\mu_{f_{A}}^{e}(x)$.

Definition 2.17. [19, 24] A fuzzy soft point $x_{\alpha}^{e}$ is said to be a quasi-coincident with a fuzzy soft set $f_{A}$, denoted by $x_{\alpha}^{e}$ $q f_{A}$, if $\alpha+\mu_{f_{A}}^{e}(x)>1$. Otherwise, $x_{\alpha}^{e}$ is non-quasi-coincident with $f_{A}$ and denoted by $x_{\alpha}^{e} \bar{q} f_{A^{*}}$

Definition 2.18. [19, 24] A fuzzy soft set $f_{A}$ is said to be quasi-coincident with $g_{B}$, denoted by $f_{A} q g_{B}$, if there exists $x \in X$ such that $\mu_{f_{A}}^{e}(x)+\mu_{g_{B}}^{e}(x)>1$, for some $e \in A \cap B$. If this is true we can say that $f_{A}$ and $g_{B}$ are quasicoincident at $x$.

Proposition 2.1. $[19,24]$ Let $f_{A}$ and $g_{B}$ be two fuzzy soft sets, $f_{A} \subseteq g_{B}$ if and only if $f_{A} \bar{q}\left(g_{B}\right)^{c}$. In particular, $x_{\alpha}^{B}$ $\widetilde{E} f_{A}$ if and only if $x_{\alpha}^{e} \bar{q}\left(f_{A}\right)^{c}$.

Definition 2.19. [11] Let $(X, \tau, E)$ be a fuzzy soft topological space and $g_{B}$ be a fuzzy soft subset of $X$. Then $\tau_{g_{B}}$ $=\left\{g_{B} \tilde{\cap} f_{A} ; f_{A} \in \tau\right\}$ is called fuzzy soft relative topology and $\left(g_{B}, \tau_{g_{B}}, B\right)$ is called fuzzy soft subspace. If $g_{B} \in \tau$, then $\left(g_{B}, \tau_{g_{B}}, B\right)$ is called fuzzy soft open subspace. If $g_{B} \in \tau^{\circ}$, then $\left(g_{B}, \tau_{g_{B}}, B\right)$ is called fuzzy soft closed subspace.

Lemma 2.1. [11] Let $(X, \tau, E)$ be a fuzzy soft topological space on $X$ and $g_{B} \subseteq f_{A} \in F S S(X)_{E}$. Then, $F C l_{f_{A}}\left(g_{B}\right)=\operatorname{Fcl}\left(g_{B}\right) \tilde{\cap} f_{A}$.

Definition 2.19. [17] Let $F S S(X)_{E}$ and $F S S(Y)_{K}$ be families of fuzzy soft sets over $X$ and $Y$, respectively. Let $u: X \rightarrow Y$ and $p: E \rightarrow K$ be mappings. Then the map $f_{p u}$ is called fuzzy soft mapping from $\operatorname{FSS}(X)_{E}$ to $\operatorname{FSS}(Y)_{K}$, denoted by $f_{p u}: F S S(X)_{E} \rightarrow F S S(Y)_{K}$, such that:

(1) If $g_{B} \in F S S(X)_{E}$, then the image of $g_{B}$ under the fuzzy soft mapping $f_{p u}$ is a fuzzy soft set over $Y$ defined by $f_{p u}\left(g_{B}\right)$ where $\forall k \in p(E), \forall y \in Y$,

$f_{p u}\left(g_{B}\right)(k)(y)= \begin{cases}\underset{u(x)=y}{\vee}\left[\underset{p(e)=k}{\vee}\left(g_{B}(e)\right)\right](x) & \text { if } x \in u^{-1}(y) \\ 0 & \text { otherwise }\end{cases}$

(2) If $h_{C} \in F S S(Y)_{K}$, then the pre-image of $h_{C}$ under the fuzzy soft mapping $f_{p u}, f_{p u}^{-1}\left(h_{C}\right)$ is a fuzzy soft set over $X$ defined by $\forall e \in p^{-1}(K), \forall x \in X$,

$$
f_{p u}^{-1}\left(h_{C}\right)(e)(x)=\left\{\begin{array}{cc}
h_{C}(p(e))(u(x)) & \text { for } p(e) \in C \\
0 & \text { otherwise }
\end{array}\right.
$$

Definition 2.20. [17] The fuzzy soft mapping $f_{p u}$ is called surjective (respectively, injective) if $p$ and $u$ are surjective (respectively, injective), also $f_{p u}$ is said to be constant if $p$ and $u$ are constant.

Definition 2.21. [17] Let $\left(X, \tau_{1}, E\right)$ and $\left(Y, \tau_{2}, K\right)$ be two fuzzy soft topological spaces and $f_{p u}: F S S(X)_{E} \rightarrow$ $\operatorname{FSS}(Y)_{K}$ be a fuzzy soft mapping. Then $f_{p u}$ is called: 
(1) Fuzzy soft continuous if $f_{p u}^{-1}\left(h_{C}\right) \in \tau_{1} \forall h_{C} \in \tau_{2}$.

(2) Fuzzy soft open if $f_{p u}\left(g_{B}\right) \in \tau_{2} \forall g_{B} \in \tau_{1}$.

Definition 2.22. [9] Two non-null fuzzy soft sets $f_{E}$ and $g_{E}$ are said to be fuzzy soft $Q$-separated in a fuzzy soft topological space $(X, \tau, E)$ if $\operatorname{Fcl}\left(f_{E}\right) \tilde{\cap} g_{E}=f_{E} \tilde{\cap} \operatorname{Fcl}\left(g_{E}\right)=\widetilde{0}_{E}$.

Theorem 2.1. [9] Let $(X, \tau, E)$ be a fuzzy soft topological space, $f_{E}$ and $g_{E}$ be two fuzzy soft closed sets in $X$. If $f_{E} \tilde{\cap} g_{E}$, then $f_{E}$ and $g_{E}$ are fuzzy soft $Q$-separated sets.

Theorem 2.2. [9] Let $(X, \tau, E)$ be a fuzzy soft topological space and $f_{E} \in F S S(X)_{E} \cdot f_{E}$ is called $F S C_{M}$ connected if and only if it cannot be written as a union of fuzzy soft $Q$-separated sets.

Theorem 2.3. [9] A fuzzy soft topological space $(X, \tau, E)$ is $F S C_{M}$-connected if and only if $\tilde{1}_{E}$ cannot be written as a union of fuzzy soft $Q$-separated sets.

Theorem 2.4. [9] Let $(X, \tau, E)$ be a fuzzy soft topological space and $f_{E} \in F S S(X)_{E}$ be an open $F S C_{M^{-}}$-connected set in $X$. If $f_{E} \subseteq g_{E} \subseteq F c l\left(f_{E}\right)$, then $g_{E}$ is a $F S C_{M}$-connected set.

Remark 2.1. [9] Let $(X, \tau, E)$ be a fuzzy soft topological space and $f_{E} \in F S S(X)_{E}$ be an open $F S C_{M}$-connected set in $X$. Then $F C l\left(f_{E}\right)$ is a $F S C_{M}$-connected set.

Definition 2.23. [9] Let $(X, \tau, E)$ be a fuzzy soft topological space and $f_{E} \in \operatorname{FSS}(X)_{E}$. Then, $f_{E}$ is called:

(1) $F S C_{1}$-connected: if does not exist two non-null fuzzy soft open sets $h_{E}$ and $s_{E}$ such that

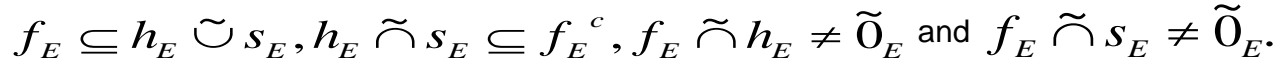

(2) FSC $_{2}$-connected: if does not exist two non-null fuzzy soft open sets $h_{E}$ and $s_{E}$ such that $f_{E} \subseteq h_{E} \widetilde{\cup} s_{E}, h_{E} \tilde{\curvearrowright} h_{E} \tilde{\curvearrowright} s_{E}=\widetilde{\mathrm{O}}_{E}, f_{E} \tilde{\cap} h_{E} \neq \widetilde{\mathrm{O}}_{E}$ and $f_{E} \tilde{\curvearrowright} s_{E} \neq \widetilde{\mathrm{O}}_{E}$.

(3) $F_{S} C_{3}$-connected: if does not exist two non-null fuzzy soft open sets $h_{E}$ and $s_{E}$ such that $f_{E} \subseteq h_{E} \sim s_{E}, h_{E} \widetilde{\curvearrowright} s_{E} \subseteq f_{E}^{c}, h_{E} \not \subset f_{E}^{c}$ and $s_{E} \not \subset f_{E}^{c}$.

(4) $F S C_{4}$-connected: if does not exist two non-null fuzzy soft open sets $h_{E}$ and $s_{E}$ such that

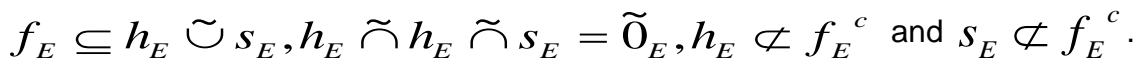

Otherwise, $f_{E}$ is called $F S C_{i}$-disconnected set for $i=1,2,3,4$.

In the above definition, if we take $\tilde{1}_{E}$ instead of $f_{E}$, then the fuzzy soft topological space $(X, \tau, E)$ is called $F S C_{i-}$ connected space $(i=1,2,3,4)$.

Remark 2.2. [9] The relationship between $F S C_{i}$-connectedness $(i=1,2,3,4)$ can be described by the following diagram:

$$
\begin{array}{lll}
\mathrm{FSC}_{1} & \Rightarrow & \mathrm{FSC}_{2} \\
\Downarrow & \Downarrow \\
\mathrm{FSC}_{3} & \Rightarrow & \mathrm{FSC}_{4}
\end{array}
$$

Remark 2.3. [9] The reverse implications is not true in general (see Examples 3.14, 3.15, 3.16, 3.17 in [9]). But example 3.17 in [9] is incorrect, we must take $\mu_{g(b)}(x)=\frac{2}{3}$ if $\frac{2}{3}<x \leq 1$.

Theorem 2.5. [9] Let $f_{\bar{\nabla} u}:(X, \tau, E) \rightarrow(Y, \sigma, K)$ be a fuzzy soft surjective continuous mapping and $f_{E} \in F S S(X)_{E}$. If $f_{E}$ is a $F S C_{i}$-connected set in $X$, then $f_{p u}\left(f_{E}\right)$ is a $F S C_{i}$-connected set in $Y$ for $i=1,2,3,4$.

\section{FUZZY SOFT SEPARATED SETS IN FUZZY SOFT TOPOLOGICAL SPACES}

In this section, we will introduce different notions of fuzzy soft separated sets and study the relation between these 
notions. Also, we will investegate the characterizations of the fuzzy soft separated sets.

Definition 3.1. Two non-null fuzzy soft sets $f_{E}, g_{E}$ are said to be fuzzy soft weakly separated in a fuzzy soft topological space $(X, \tau, E)$ if $F c l\left(f_{E}\right) \bar{q} g_{E}$ and $f_{E} \bar{q} \operatorname{Fcl}\left(g_{E}\right)$.

Theorem 3.1. Let $(X, \tau, E)$ be a fuzzy soft topological space and $f_{E^{\prime}} g_{E} \in F S S(X)_{E}$. Then, $f_{E}$ and $g_{E}$ are fuzzy soft weakly separated sets iff there exist fuzzy soft open sets $h_{E}$ and $s_{E}$ such that $f_{E} \subseteq h_{E}, g_{E} \subseteq s_{E}, f_{E} \bar{q} s_{E}$, and $g_{E} \bar{q} h_{E}$

Proof. Let $f_{E}, g_{E}$ be fuzzy soft weakly separated sets in $\left(X_{,} \tau_{,} E\right)$. Then $F c l\left(f_{E}\right) \bar{q} g_{E}$ and $f_{E} \bar{q} F c l\left(g_{E}\right)$. Therefore, $g_{E} \subseteq\left[\mathrm{Fcl}\left(f_{E}\right)\right]^{c}$ and $f_{E} \subseteq\left[\mathrm{Fcl}\left(g_{E}\right)\right]^{c}$. Taking $h_{E}=\left[\mathrm{Fcl}\left(g_{E}\right)\right]^{c}$ and $s_{E}=\left[\mathrm{Fcl}\left(f_{E}\right)\right]^{c}$. Then $h_{E}, s_{E} \in \tau, f_{E} \bar{q} s_{E}$, and $g_{E} \bar{q} h_{E}$. The converse is obvious.

Remark 3.1. If $f_{E}$ and $g_{E}$ are fuzzy soft $Q$-separated, then $f_{E}$ and $g_{E}$ are fuzzy soft weakly separated.

Proof. The result follows from Definitions 3.1, 2.22.

Remark 3.2. If $f_{E}$ and $g_{E}$ are fuzzy soft weakly separated, then they may not be fuzzy soft $Q$-separated as shown by the following example.

Example 3.1. Let $X=\{a, b\}, E=\left\{e_{1}, e_{2}\right\}, A=\left\{e_{1}\right\} \subseteq E$ and $\tau=\left\{\tilde{1}_{E}, \tilde{0}_{E},\left\{\left(e_{1},\left\{a_{0.3}, b_{0.2}\right\}\right),\left(e_{2},\left\{a_{0.5}\right.\right.\right.\right.$, $\left.\left.\left.\left.b_{0.3}\right\}\right)\right\}\right\}$ be a fuzzy sof topology on $X$. If $f_{A}=\left\{\left(e_{1},\left\{a_{0.1}\right\}\right)\right\}$ and $g_{A}=\left\{\left(e_{1},\left\{a_{0.1}, b_{0.1}\right\}\right)\right\}$, then $f_{A}$ and $g_{A}$ are fuzzy soft weakly separated sets. But $f_{A}$ and $g_{A}$ are not fuzzy soft $Q$-separated.

Definition 3.2. Two non-null fuzzy soft sets $f_{E}$ and $g_{E}$ are said to be fuzzy soft separated in a fuzzy soft topological space $(X, \tau, E)$ if there exist non-null fuzzy soft open sets $h_{E}$ and $s_{E}$ such that $f_{E} \subseteq h_{E}, g_{E} \subseteq s_{E}$ and $f_{E} \tilde{\cap} s_{E}=g_{E} \tilde{\cap} h_{E}=\widetilde{0}_{E}$.

Remark 3.3. If $f_{E}$ and $g_{E}$ are fuzzy soft separated sets, then $f_{E}$ and $g_{E}$ are fuzzy soft weakly separated sets.

Proof. Let $f_{E}$ and $g_{E}$ be fuzzy soft separated sets. Then, there exist two non-null fuzzy soft open sets $h_{E}$ and $s_{E}$ such that $f_{E} \subseteq h_{E}, g_{E} \subseteq s_{E}$ and $f_{E} \tilde{\cap} s_{E}=g_{E} \widetilde{\cap} h_{E}=\widetilde{\mathrm{O}}_{E}$. Therefore, $f_{E} \bar{q} s_{E}$ and $g_{E} \bar{q} h_{E}$. By Theorem 3.1, $f_{E}$ and $g_{E}$ are fuzzy soft weakly separated sets.

Remark 3.4. If $f_{E}$ and $g_{E}$ are fuzzy soft weakly separated, then they may not be fuzzy soft separated. In fact, $f_{A}$ and $g_{A}$ defined in Example 3.1 are fuzzy soft weakly separated, but they are not fuzzy soft separated.

Remark 3.5. The notions of fuzzy soft separated sets and fuzzy soft $Q$-separeted sets are independent of each other as shown by the following examples.

Example3.2. Let $X=\{a, b\}, E=\{e\}$ and

$\tau=\left\{\tilde{1}_{E}, \widetilde{0}_{E}, h_{E}=\left\{\left(e,\left\{a_{0.5}\right\}\right)\right\}, s_{E}=\left\{\left(e,\left\{b_{0.5}\right\}\right)\right\}, h_{E} \widetilde{\cup}_{E}\right\} \quad$ be $\quad$ a fuzzy soft topology on $X$. If $f_{E}=\left\{\left(e_{,}\left\{a_{0.1}\right\}\right)\right\}$ and $g_{E}=\left\{\left(e_{,}\left\{b_{0,1}\right\}\right)\right\}$, then there exist two non-null fuzzy soft open sets $h_{E}$ and $s_{E}$ such that $f_{E} \subseteq h_{E}, g_{E} \subseteq s_{E}$ and $f_{E} \tilde{\cap} s_{E}=g_{E} \tilde{\cap} h_{E}=\widetilde{0}_{E}$. So, $f_{E}$ and $g_{E}$ are fuzzy soft separated sets. But $f_{E}$ and $g_{E}$ are not fuzzy soft $Q$-separated sets since, $\operatorname{Fcl}\left(f_{E}\right)=h_{E} \widetilde{\sim} s_{E}$ and $\operatorname{Fcl}\left(f_{E}\right) \tilde{\cap} g_{E} \neq \widetilde{0}_{E}$.

Example 3.3. Let $X=\{a, b\}, E=\{e\}$ and

$\tau=\left\{\tilde{1}_{E}, \widetilde{0}_{E}, h_{E}=\left\{\left(e,\left\{a_{1}, b_{0.1}\right\}\right)\right\}, s_{E}=\left\{\left(e,\left\{a_{0.1}, b_{1}\right\}\right)\right\}, h_{E} \widetilde{\cup}_{s_{E}}\right\}$ be a fuzzy soft topology on $X$. If $f_{E}=\left\{\left(e_{,}\left\{a_{0.6}\right\}\right)\right\}$ and $g_{E}=\left\{\left(e_{,}\left\{b_{0.7}\right\}\right)\right\}$, then $f_{E}$ and $g_{E}$ are fuzzy soft $Q$-separated sets. But $f_{E} \widetilde{\cap} s_{E} \neq \widetilde{0}_{E}$ and $g_{E} \widetilde{\cap} h_{E} \neq \widetilde{0}_{E}$. So, $f_{E}$ and $g_{E}$ are not fuzzy soft separated sets.

Definition 3.3. Let $f_{E} \in F S S(X)_{E}$. The support of $f_{E}(e)$, denoted by $S\left(f_{E}(e)\right)$, is the set, $S\left(f_{E}(e)\right)=\left\{x \in X_{;} f_{E}(e)(x)>0\right\}$.

Definition 3.4. Two fuzzy soft sets $f_{E}$ and $g_{E}$ are said to be quasi-coincident with respect to $f_{E}$ if $\mu_{f_{E}}^{e}(x)+\mu_{g_{E}}^{e}(x)>1$ for every $x \in S\left(f_{E}(e)\right)$. 
Definition 3.5. Two non-null fuzzy soft sets $f_{E}$ and $g_{E}$ are said to be fuzzy soft strongly separated in a fuzzy soft topological space $(X, \tau, E)$ if there exist $h_{E}$ and $s_{E} \in \tau$ such that $f_{E} \subseteq h_{E}, g_{E} \subseteq s_{E}$, $f_{E} \widetilde{\cap} s_{E}=g_{E} \tilde{\cap} h_{E}=\widetilde{\mathrm{O}}_{E}, f_{E}$ and $h_{E}$ are fuzzy soft quasi-coincident with respect to $f_{E}$, and $g_{E}$ and $s_{E}$ are fuzzy soft quasi-coincident with respect to $g_{E}$.

Remark 3.6. If $f_{E}$ and $g_{E}$ are fuzzy soft strongly separated, then $f_{E}$ and $g_{E}$ are fuzzy soft separated and fuzzy soft weakly separated.

Proof. The result follows from Definitions 3.5, 3.2 and Remark 3.3.

Remark 3.7. If $f_{E}$ and $g_{E}$ are fuzzy soft separated, then they may not be fuzzy soft strongly separated as shown by the following example.

Example 3.4. Let $X=\{a, b\}, E=\left\{e_{1}, e_{2}\right\}, A=\left\{e_{1}\right\}, B=\left\{e_{2}\right\} \subseteq E$ and $\tau=\left\{\tilde{1}_{E}, \tilde{0}_{E},\left\{\left(e_{1},\left\{a_{0.3}\right.\right.\right.\right.$, $\left.\left.\left.\left.b_{0.2}\right\}\right)\right\},\left\{\left(e_{2},\left\{a_{0.2}, b_{0.2}\right\}\right)\right\},\left\{\left(e_{1},\left\{a_{0.3}, b_{0.2}\right\}\right),\left(e_{2},\left\{a_{0.2}, b_{0.2}\right\}\right)\right\}\right\}$ be a fuzzy soft topology on $X$. Let $f_{A}=\left\{\left(e_{1},\left\{a_{0.1}\right\}\right)\right\}$ and $g_{B}=\left\{\left(e_{2},\left\{b_{0.2}\right\}\right)\right\}$. Then, $f_{A}$ and $g_{B}$ are fuzzy soft separated sets, but $f_{A}$ and $g_{B}$ are not fuzzy soft strongly separated.

Remark 3.8. The notions of fuzzy soft $Q$-separated and fuzzy soft strongly separated are independent to each others as shown by the following examples:

Example 3.5. Let $X=\{a, b\}, E=\left\{e_{1}, e_{2}\right\}, A=\left\{e_{1}\right\}, B=\left\{e_{2}\right\} \subseteq E$ and $\tau=\left\{\tilde{1}_{E}, \tilde{0}_{E},\left\{\left(e_{1},\left\{a_{0.7}\right.\right.\right.\right.$, $\left.\left.\left.\left.b_{0.2}\right\}\right)\right\},\left\{\left(e_{2},\left\{a_{0.2}, b_{0.7}\right\}\right)\right\},\left\{\left(e_{1},\left\{a_{0.7}, b_{0.2}\right\}\right),\left(e_{2},\left\{a_{0.2}, b_{0.7}\right\}\right)\right\}\right\}$ be a fuzzy soft topology on $X$. Let $f_{A}=\left\{\left(e_{1},\left\{a_{0.5}\right\}\right)\right\}$ and $g_{B}=\left\{\left(e_{2},\left\{b_{0.4}\right\}\right)\right\}$. Then, $f_{A}$ and $g_{B}$ are fuzzy soft strongly separated sets, but $f_{A}$ and $g_{B}$ are not fuzzy soft $Q$-separated.

Example 3.6. Let $X=\{a, b\}, E=\left\{e_{1}, e_{2}\right\}, A=\left\{e_{1}\right\}, B=\left\{e_{2}\right\} \subseteq E$ and $\tau=\left\{\tilde{1}_{E}, \tilde{0}_{E},\left\{\left(e_{1},\left\{a_{0.3}, b_{0.2}\right\}\right)\right.\right.$, $\left.\left.\left(e_{2},\left\{a_{1}, b_{1}\right\}\right)\right\},\left\{\left(e_{1},\left\{a_{1}, b_{1}\right\}\right),\left(e_{2},\left\{a_{0.1}, b_{0.4}\right\}\right)\right\},\left\{\left(e_{1},\left\{a_{0.3}, b_{0.2}\right\}\right),\left(e_{2},\left\{a_{0.1}, b_{0.4}\right\}\right)\right\}\right\}$ be a fuzzy soft topology on $X$. Let $f_{A}=\left\{\left(e_{1},\left\{a_{0.2}\right\}\right)\right\}$ and $g_{B}=\left\{\left(e_{2},\left\{b_{0.3}\right\}\right)\right\}$. Then, $f_{A}$ and $g_{B}$ are fuzzy soft $Q$-separated sets, but $f_{A}$ and $g_{B}$ are not fuzzy soft strongly separated.

Remark 3.9. In fuzzy soft topological space $(X, \tau, E)$ the relationship between different notions of fuzzy soft separated sets can be discribed by the following diagram.

\section{fuzzy soft strongly separated \\ $\Downarrow$}

fuzzy soft separated

$\Downarrow$

fuzzy soft $Q$ - separated $\Rightarrow$ fuzzy soft weakly separated

Theorem 3.2. Let $f_{E}$ and $g_{E}$ be fuzzy soft $Q$-separated (respectively, separated, strongly separated, weakly separated) sets in $X$ and $h_{E} \subseteq f_{E}, s_{E} \subseteq g_{E}$. Then, $h_{E}$ and $s_{E}$ are fuzzy soft $Q$-separated (respectively, separated, strongly separated, weakly separated) sets in $X$.

Proof. As a sample, we will prove the case fuzzy soft $Q$-sepaeated. Let $f_{E}$ and $g_{E}$ be fuzzy soft $Q$-separated sets in $X$. Then, $\quad F c l\left(f_{E}\right) \tilde{\cap} g_{E}=f_{E} \tilde{\cap} F c l\left(g_{E}\right)=\widetilde{0}_{E}$. Since $\quad h_{E} \subseteq f_{E} \quad$ and $\quad s_{E} \subseteq g_{E}$, then $\operatorname{Fcl}\left(h_{E}\right) \tilde{\cap} s_{E}=h_{E} \tilde{\cap} \operatorname{Fcl}\left(s_{E}\right)=\tilde{0}_{E}$. Therefore, $h_{E}$ and $s_{E}$ are fuzzy soft $Q$-separated sets in $X$.

Theorem 3.3. Let $(X, \tau, E)$ be a fuzzy soft topological space and $f_{E}, g_{E} \in F S S(X)_{E}$. Then, $f_{E}$ and $g_{E}$ are fuzzy soft $Q$-separated in $X$ if there are fuzzy soft closed sets $h_{E}$ and $s_{E}$ such that $f_{E} \subseteq h_{E}, g_{E} \subseteq s_{E}$, and $f_{E} \tilde{\cap} s_{E}=g_{E} \tilde{\cap} h_{E}=\widetilde{0}_{E}$.

Proof. Let $f_{E}$ and $g_{E}$ be fuzzy soft $Q$-separated in $X$. Then $F c l\left(f_{E}\right) \tilde{\cap} g_{E}=f_{E} \tilde{\cap} F c l\left(g_{E}\right)=\tilde{0}_{E}$. Taking $h_{E}=\operatorname{Fcl}\left(f_{E}\right)$ and $s_{E}=\operatorname{Fcl}\left(g_{E}\right)$. Therefore, $h_{E}$ and $s_{E}$ are fuzzy soft closed sets in $X$ such that $f_{E} \subseteq h_{E}$, $g_{E} \subseteq s_{E}$, and $f_{E} \widetilde{\cap} s_{E}=g_{E} \widetilde{\cap} h_{E}=\widetilde{0}_{E}$. 
Theorem 3.4. Let $(X, \tau, E)$ be a fuzzy soft topological space and $g_{E} \subseteq f_{E} \in F S S(X)_{E}$. Two fuzzy soft sets $h_{E}$ and $s_{E}$ are fuzzy soft separated (respectively, $Q$ - sepaeated, strongly separated) in $\left(g_{E}, \tau_{g_{E}}, E\right)$ if and only if $h_{E}, s_{E}$ be fuzzy soft separated $\left(Q\right.$ - sepaeated, strongly separated, respectively) in $\left(f_{E}, \tau_{f_{E}}, E\right)$.

Proof. As a sample, we will prove the case fuzzy soft $Q$ - sepaeated. Let $h_{E}$ and $s_{E}$ be fuzzy soft $Q$ - sepaeated in $\left(g_{E}\right.$, $\tau_{g_{E}}$, $\quad E$ ). Then $F c l_{g_{E}}\left(h_{E}\right) \widetilde{\cap} s_{E}=h_{E} \tilde{\cap} F c l_{g_{E}}\left(s_{E}\right)=\widetilde{O}_{E}$. Since $\quad g_{E} \subseteq f_{E}$, then $F c l_{g_{E}}\left(h_{E}\right)=F c l_{f_{E}}\left(h_{E}\right) \tilde{\cap} g_{E} \quad$ and $\quad F c l_{g_{E}}\left(s_{E}\right)=F c l_{f_{E}}\left(s_{E}\right) \tilde{\cap} g_{E}$. Therefore, $F c l_{f_{E}}\left(h_{E}\right) \widetilde{\cap} s_{E}=h_{E} \tilde{\cap} F c l_{f_{E}}\left(s_{E}\right)=\widetilde{\mathrm{O}}_{E}$. Hence, $h_{E}$ and $s_{E}$ are fuzzy soft $Q$ - sepaeated in $\left(f_{E}, \tau_{f_{E}}, E\right)$.

Conversely, Let $h_{E}$ and $s_{E}$ be fuzzy soft $Q$ - sepaeated in $\left(f_{E}, \tau_{f_{E}}, \quad E\right)$. Then $F c l_{f_{E}}\left(h_{E}\right) \tilde{\cap} s_{E}=h_{E} \tilde{\cap} F c l_{f_{E}}\left(s_{E}\right)=\widetilde{0}_{E} . \quad$ Therefore, $\quad\left(F c l_{f_{E}}\left(h_{E}\right) \tilde{\cap} g_{E}\right) \tilde{\cap} s_{E}$ $=h_{E} \tilde{\cap}\left(F_{c l} l_{f_{E}}\left(s_{E}\right) \tilde{\cap} g_{E}\right)=\widetilde{0}_{E}$. And so, $F c l_{g_{E}}\left(h_{E}\right) \tilde{\cap} s_{E}=h_{E} \tilde{\cap} F c l_{g_{E}}\left(s_{E}\right)=\tilde{0}_{E}$. Hence, $h_{E}$ and $s_{E}$ are fuzzy soft $Q$ - sepaeated in $\left(g_{E}, \tau_{g_{E}}, E\right)$.

Theorem 3.5. Let $(X, \tau, E)$ be a fuzzy soft topological space and $g_{E} \subseteq f_{E} \in F S S(X)_{E}$. If $h_{E}$ and $s_{E}$ are fuzzy soft weakly separated sets in $\left(f_{E}, \tau_{f_{E}}, E\right)$, then $h_{E}$ and $s_{E}$ are fuzzy soft weakly separated in $\left(g_{E}, \tau_{g_{E}}, E\right)$.

Proof. Let $h_{E}$ and $s_{E}$ be fuzzy soft weakly separated sets in $\left(f_{E}, \tau_{f_{E}}, E\right)$. Then $F_{c l} l_{\tau_{f E}}\left(h_{E}\right) \bar{q} s_{E}$ and $h_{E} \bar{q}$ $F_{c l} l_{\tau_{f E}}\left(s_{E}\right)$. Since $g_{E} \subseteq f_{E}$, then $F c l_{\tau_{g_{E}}}\left(h_{E}\right)=F c l_{\tau_{f_{E}}}\left(h_{E}\right) \tilde{\cap} g_{E} \subseteq F c l_{\tau_{f_{E}}}\left(h_{E}\right)$ and $F c l_{\tau_{g_{E}}}\left(s_{E}\right)=F c l_{\tau_{f_{E}}}\left(s_{E}\right) \tilde{\cap} g_{E} \subseteq F c l_{\tau_{f E}}\left(s_{E}\right)$. Therefore, $F c l_{\tau g_{E}}\left(h_{E}\right) \bar{q} s_{E}$ and $h_{E} \bar{q} F c l_{\tau g_{E}}\left(s_{E}\right)$. Thus, $h_{E}$ and $s_{E}$ be fuzzy soft weakly separated sets in $\left(g_{E}, \tau_{g_{E}}, E\right)$.

Remark 3.10. The converse of Theorem 3.5 is not true in general as shown by the following example:

Example 3.7. Let $X=\{a, b\}, E=\left\{e_{1}, e_{2}\right\}, A=\left\{e_{1}\right\} \subseteq E$ and $\tau_{0}=\left\{\tilde{1}_{E}, \tilde{0}_{E}\right\}$ be the fuzzy soft indiscrete topology on $X$. If $h_{A}=\left\{\left(e_{1},\left\{a_{0.1}, b_{0.2}\right\}\right)\right\}$ and $s_{A}=\left\{\left(e_{1},\left\{a_{0.1}, b_{0.3}\right\}\right)\right\} \subseteq f_{E}=\left\{\left(e_{1},\left\{a_{0.1}, b_{0.3}\right\}\right),\left(e_{2}\right.\right.$, $\left.\left.\left\{a_{0.3}, b_{0.1}\right\}\right)\right\}$. Then $h_{A}$ and $s_{\square}$ are fuzzy soft weakly separated sets in $\left(f_{E}, \tau_{f_{E}}, E\right)$ but $h_{A}$ and $s_{A}$ are not fuzzy soft weakly separated sets in $(X, \tau, E)$.

\section{FUZZY SOFT CONNECTED SETS IN FUZZY SOFT TOPOLOGICAL SPACES}

In this section, we introduce different notions of connectedness of fuzzy soft sets and study the relation between these notions. Also, we will investegate the characterizations of the fuzzy soft connected sets..

Definition 4.1. A fuzzy soft set $f_{E}$ in a fuzzy soft topological space $(X, \tau, E)$ is called $F S C_{M}$-disconnected set if there exist two non-null fuzzy soft $Q$-sepaeated sets $h_{E}$ and $s_{E}$ in $X$ such that $f_{E}=h_{E} \widetilde{\cup} s_{E}$. Otherwise, $f_{E}$ is called $F S C_{M}$-connected set.

Definition 4.2. A fuzzy soft set $f_{E}$ in a fuzzy soft topological space $(X, \tau, E)$ is called $F S C_{S}$-disconnected set if there exist two non-null fuzzy soft weakly-sepaeated sets $h_{E}$ and $s_{E}$ in $X$ such that $f_{E}=h_{E} \widetilde{\cup}_{s_{E}}$. Otherwise, $f_{E}$ is called $F S C_{S}$-connected set.

Definition 4.3. A fuzzy soft set $f_{E}$ in a fuzzy soft topological space $(X, \tau, E)$ is called FSO -disconnected (respectively, $\mathrm{FSO}_{q}$-disconnected) set if there exist two non-null fuzzy soft sepaeated (respectively, strongly separated) sets $h_{E}$ and $s_{E}$ in $X$ such that $f_{E}=h_{E} \widetilde{\cup} s_{E}$. Otherwise, $f_{E}$ is called FSO -connected (respectively, FSO $O_{q}$. connected) set.

Definition 4.4. A fuzzy soft set $f_{E}$ in a fuzzy soft topological space $(X, \tau, E)$ is called $F S C_{5}$-connected set in $X$ if there does not exist any non-null proper fuzzy soft clopen set in $\left(f_{E}, \tau_{f_{E}}, E\right)$. Note that, this kind of fuzzy soft connectedness was studied by Mahanta [11].

In the above definitions, if we take $\tilde{1}_{E}$ instead of $f_{E}$, then the fuzzy soft topological space $(X, \tau, E)$ is called $F S C_{M^{-}}$ 
connected (respectively, $\mathrm{FSC}_{S}$-connected, $F S O$-connected, $\mathrm{FSO}_{\mathrm{q}}$-connected, $F S C_{5}$-connected) space.

Theorem 4.1. Let $(X, \tau, E)$ be a fuzzy soft topological space, $f_{E} \in F S S(X)_{E}$. If $f_{E}$ is a $F S C_{S}$-connected set in $X$, then $f_{E}$ is a $F S C_{M}$-connected.

Proof. Let $f_{E}$ be a $F S C_{S}$-connected set in $X$. Suppose $f_{E}$ is a $F S C_{M}$-disconnected. Then, there exist two non-null fuzzy soft $Q$-sepaeated sets $h_{E}$ and $s_{E}$ in $X$ such that $f_{E}=h_{E} \widetilde{\cup} s_{E}$. By Remark 3.1, $h_{E}$ and $s_{E}$ are non-null fuzzy soft weakly-sepaeated sets in $X$ such that $f_{E}=h_{E} \widetilde{\cup} s_{E}$. Therefore, $f_{E}$ is a $F S C_{S}$-disconnected set in $X$. This a contraduction. Hence, $f_{E}$ is a FSC $_{M}$-connected.

Remark 4.1. If $f_{E}$ is a $F S C_{M}$-connected, then it may not be a $F S C_{S}$-connected as shown by the following example.

Example 4.1. Let $X=\{a, b\}, E=\left\{e_{1}, e_{2}\right\}, A=\left\{e_{1}\right\} \subseteq E, \tau=\left\{\tilde{1}_{E}, \tilde{0}_{E},\left\{\left(e_{1},\left\{a_{0.3}, b_{0.2}\right\}\right),\left(e_{2},\left\{a_{0.5}\right.\right.\right.\right.$, $\left.\left.\left.\left.b_{0.3}\right\}\right)\right\}\right\}$ be a fuzzy soft topology on $X$ and $f_{A}=\left\{\left(e_{1},\left\{a_{0.1}, b_{0.1}\right\}\right)\right\}$. Then, there exist $h_{A}=\left\{\left(e_{1},\left\{a_{0.1}\right\}\right)\right\}$ and $s_{A}=\left\{\left(e_{1},\left\{b_{0,1}\right\}\right)\right\}$ such that $\operatorname{Fcl}\left(h_{A}\right) \bar{q} s_{A}, h_{A} \bar{q} \operatorname{Fcl}\left(s_{A}\right), f_{A}=h_{A} \widetilde{\cup}_{s_{A}}$. So, $f_{A}$ is not a $F S C_{S}$-connected. But $f_{A}$ is a $F S C_{M}$-connected.

Theorem 4.2. Let $(X, \tau, E)$ be a fuzzy soft topological space, $f_{E} \in F S S(X)_{E}$. If $f_{E}$ is a $F S C_{1}$-connected set in $X$, then $f_{E}$ is a $F S C_{S}$-connected.

Proof. Let $f_{E}$ be a $F S C_{1}$-connected set in $X$. Suppose $f_{E}$ is a $F S C_{S}$-disconnected. Then, there exist two non-null fuzzy soft weakly -sepaeated sets $h_{E}$ and $s_{E}$ in $X$ such that $f_{E}=h_{E} \widetilde{\cup} s_{E}$. By Theorem 3.1, there exist two fuzzy soft open sets $g_{E}$ and $u_{E}$ such that $h_{E} \subseteq g_{E}, s_{E} \subseteq u_{E}, h_{E} \bar{q} u_{E}$, and $s_{E} \bar{q} g_{E}$. Then, $f_{E} \subseteq g_{E} \widetilde{\cup} u_{E}$.

Also, $f_{E} \tilde{\cap} g_{E} \neq \tilde{0}_{E}$. For, if $f_{E} \tilde{\cap} g_{E}=\tilde{0}_{E}$, then $f_{E} \tilde{\cap} h_{E}=\tilde{0}_{E}$ so that $h_{E}=\tilde{0}_{E}$ (since $f_{E}=h_{E} \tilde{\cup}_{E}$ implies $h_{E} \subseteq f_{E}$ ), which contradicts that $h_{E}$ is a non-null. Similarly, $f_{E} \tilde{\cap} u_{E} \neq \tilde{0}_{E}$.

Also, $g_{E} \tilde{\cap} u_{E} \subseteq\left(f_{E}\right)^{c}$. For, if $g_{E} \tilde{\cap} u_{E} \not \subset\left(f_{E}\right)^{c}$, then there exist $x \in X, \quad e \in E$ such that $\mu_{g_{E} \tilde{\cap} u_{E}}^{e}(x)>1-\mu_{f_{E}}^{e}(x)$. This means $\mu_{g_{E}}^{e}(x)+\mu_{f_{E}}^{e}(x)>1$ and $\mu_{u_{E}}^{e}(x)+\mu_{f_{E}}^{e}(x)>1$. Since $f_{E}=h_{E}$ òs $s_{E}$, then $\mu_{u_{E}}^{e}(x)+\mu_{h_{E}}^{e}(x)>1$ or $\mu_{u_{E}}^{e}(x)+\mu_{s_{E}}^{e}(x)>1$ and $\mu_{g_{E}}^{e}(x)+\mu_{h_{E}}^{e}(x)>1$ or $\mu_{g_{E}}^{e}(x)+\mu_{s_{E}}^{e}(x)>1$. Hence, $\left(h_{E} q u_{E}\right.$ or $\left.s_{E} q u_{E}\right)$ and $\left(s_{E} q g_{E}\right.$ or $\left.g_{E} q h_{E}\right)$. This is a contradiction. So, $f_{E}$ is a $F S C_{S}$-connected.

Remark 4.2. If $f_{A}$ is a $F S C_{S}$-connected, then it may not be a $F S C_{1}$-connected as shown by the following example.

Example 4.2. Let $X=\{a, b\}, E=\left\{e_{1}, e_{2}\right\}, A=\left\{e_{1}\right\} \subseteq E, \tau=\left\{\tilde{1}_{E}, \tilde{0}_{E},\left\{\left(e_{1},\left\{a_{0.7}, b_{0.8}\right\}\right)\right\},\left\{\left(e_{1},\left\{\left(a_{0.2}\right.\right.\right.\right.\right.$, $\left.\left.\left.\left.b_{0.3}\right\}\right)\right\}\right\}$ be a fuzzy soft topology on $X$ and $f_{A}=\left\{\left(e_{1},\left\{a_{0.4}, b_{0.4}\right\}\right)\right\}$. Then, there exist two fuzzy soft open sets $h_{A}=\left\{\left(e_{1}, \quad\left\{a_{0.7}, \quad b_{0.8}\right\}\right)\right\} \quad$ and $\quad s_{A}=\left\{\left(e_{1}, \quad\left\{\left(a_{0.2}, \quad b_{0.3}\right\}\right)\right\} \quad\right.$ such that $f_{A} \subseteq h_{A} \widetilde{\cup} s_{A}, h_{A} \tilde{\cap} s_{A} \subseteq\left(f_{A}\right)^{c}, f_{A} \widetilde{\cap} h_{A} \neq \widetilde{0}_{E}$ and $f_{A} \tilde{\cap} s_{A} \neq \widetilde{0}_{E}$. So, $f_{A}$ is not a FSC -connected. If we take $g_{A}=\left\{\left(e_{1},\left\{a_{0,4}\right\}\right)\right\}$ and $u_{A}=\left\{\left(e_{1},\left\{b_{0,4}\right\}\right)\right\}$, then $\operatorname{Fcl}\left(g_{A}\right) q u_{A}$ and $\operatorname{Fcl}\left(u_{A}\right) q g_{A}$. Therefore, $g_{A}$ and $u_{A}$ are not fuzzy soft weakly separated sets. Hence, $f_{A}$ is a $F S C_{S}$-connected.

Theorem 4.3. Let $(X, \tau, E)$ be a fuzzy soft topological space, $f_{E} \in F S S(X)_{E}$. If $f_{E}$ is a $F S C_{S}$-connected set in $X$, then $f_{E}$ is a $F S C_{2}$-connected.

Proof. Let $f_{E}$ be a $F S C_{S}$-connected set in $X$. Suppose $f_{E}$ is a $F S C_{2}$-disconnected. Then, there exist $h_{E}$ and $s_{E} \in \tau$ such that $f_{E} \subseteq h_{E} \widetilde{\cup} s_{E}, f_{E} \widetilde{\cap} h_{E} \widetilde{\cap} s_{E}=\widetilde{0}_{E}, f_{E} \widetilde{\cap} h_{E} \neq \widetilde{0}_{E} \quad$ and $\quad f_{E} \widetilde{\cap} s_{E} \neq \widetilde{0}_{E}$. Then, $f_{E}=g_{E} \widetilde{\cup} u_{E}$ where $g_{E}=f_{E} \widetilde{\cap} h_{E} \subseteq h_{E}$ and $u_{E}=f_{E} \widetilde{\cap} s_{E} \subseteq s_{E}$.

Since $f_{E} \tilde{\cap} h_{E} \tilde{\cap} s_{E}=\widetilde{0}_{E}$ and $g_{E} \subseteq h_{E}$, then $f_{E} \tilde{\cap} g_{E} \tilde{\cap} s_{E}=\widetilde{0}_{E}$. Also, since $g_{E} \subseteq f_{E}$, then $g_{E} \tilde{\cap} s_{E}=\widetilde{0}_{E}$. Therefore, $g_{E} \bar{q} s_{E}$. Similarly, $u_{E} \bar{q} h_{E}$. Hence, $f_{E}$ is not a $F S C_{S}$-connected. This complete the 
proof.

Theorem 4.4. Let $(X, \tau, E)$ be a fuzzy soft topological space, $f_{E} \in F S S(X)_{E}$. If $f_{E}$ is a $F S C_{S}$-connected set in $X$, then $f_{E}$ is a $F S C_{3}$-connected.

Proof. Let $f_{E}$ be a $F S C_{S}$-connected set in $X$. Suppose $f_{E}$ is a $F S C_{3}$-disconnected. Then, there exist two fuzzy soft open sets $h_{E}$ and $s_{E}$ such that $f_{E} \subseteq h_{E} \tilde{\cup} s_{E}, h_{E} \tilde{\cap} s_{E} \subseteq\left(f_{E}\right)^{c}, h_{E} \not \subset\left(f_{E}\right)^{c}$ and $s_{E} \not \subset\left(f_{E}\right)^{c}$. Then $f_{E}=g_{E} \widetilde{\cup} u_{E}$ where $g_{E}=f_{E} \tilde{\cap} h_{E} \subseteq h_{E}$ and $u_{E}=f_{E} \tilde{\cap} s_{E} \subseteq s_{E}$. Let $v_{E}$ and $j_{E} \in F S S(X)_{E}$ defined by:

$\mu_{v_{E}}^{e}(x)=\left\{\begin{array}{lr}\mu_{g_{E}}^{e}(x) & \text { if } \mu_{h_{E}}^{e}(x) \geq \mu_{s_{E}}^{e}(x), \\ 0 & \text { otherwise }\end{array}\right.$
$\mu_{j_{E}}^{e}(x)=\left\{\begin{array}{lr}\mu_{v_{E}}^{e}(x) & \text { if } \mu_{s_{E}}^{e}(x)>\mu_{h_{E}}^{e}(x), \\ 0 & \text { otherwise }\end{array}\right.$

Then $f_{E}=v_{E} \sim j_{E}$.

Now, $\mu_{v_{E}}^{e}(x) \neq 0$. For, $\mu_{v_{E}}^{e}(x)=0$. Since $h_{E} \not \subset\left(f_{E}\right)^{c}$, then there exist $x \in X$, $e \in E$ such that $\mu_{h_{E}}^{e}(x)+\mu_{f_{E}}^{e}(x)>1$. Then $\mu_{h_{E}}^{e}(x)>\mu_{s_{E}}^{e}(x)$. For, $\mu_{h_{E}}^{e}(x) \leq \mu_{s_{E}}^{e}(x)$ implies $\mu_{s_{E}}^{e}(x)+\mu_{f_{E}}^{e}(x)>1$ and hence $\mu_{h_{E} \widetilde{n} s_{E}}^{e}(x)>1-\mu_{f_{E}}^{e}(x)$ this is a contradiction with $h_{E} \widetilde{\cap} s_{E} \subseteq\left(f_{E}\right)^{c}$. So, $\mu_{v_{E}}^{e}(x) \neq 0$. Similarly, $\mu_{j_{E}}^{e}(x) \neq 0$.

Also, $v_{E} \subseteq g_{E} \subseteq h_{E}$ and $j_{E} \subseteq u_{E} \subseteq s_{E}$. Now, $v_{E} \bar{q} s_{E}$. For, if $v_{E} q s_{E}$, then there exist $x \in X, e \in E$ such that $\mu_{v_{E}}^{e}(x)+\mu_{s_{E}}^{e}(x)>1$ and hence $\mu_{v_{E}}^{e}(x)>0$. This means $\mu_{h_{E}}^{e}(x) \geq \mu_{s_{E}}^{e}(x)$ and so $\mu_{f_{E}}^{e}(x)=\mu_{g_{E}}^{e}(x)$, implying $\mu_{f_{E}}^{e}(x)+\mu_{h_{E}}^{e}(x)>1$ and thus $\mu_{h_{E} \widetilde{n} s_{E}}^{e}(x)>1-\mu_{f_{E}}^{e}(x)$ which is a contradiction with $h_{E} \tilde{\cap} s_{E} \subseteq\left(f_{E}\right)^{c}$. Similarly, $j_{E} \bar{q} h_{E}$. Thus, $v_{E}$ and $j_{E}$ are fuzzy soft weakly separated and $f_{E}=v_{E} \tilde{\cup} j_{E}$. So, $f_{E}$ is not a $F S C_{S}$-connected. This a contradiction. Then $f_{E}$ is a $F S C_{3}$-connected.

Remark 4.3. If $f_{E}$ is a $F S C_{3}$-connected (respectively, $C_{2}$-connected) set, then it may not be a $F S C_{S}$-connected as shown by the following example.

Example 4.3. Let $X=\{a, b\}, E=\left\{e_{1}, e_{2}\right\}, A=\left\{e_{1}\right\} \subseteq E, \tau=\left\{\tilde{1}_{E}, \tilde{0}_{E},\left\{\left(e_{1},\left\{a_{\frac{1}{g}}, b_{z}\right\}\right)\right\},\left\{\left(e_{1},\left\{a_{\frac{z}{g}}\right.\right.\right.\right.$, $\left.\left.\left.\left.b_{\frac{1}{a}}\right\}\right)\right\},\left\{\left(e_{1},\left\{a_{\frac{1}{a}}, b_{\frac{1}{a}}\right\}\right)\right\},\left\{\left(e_{1},\left\{a_{\frac{a}{a}}, b_{\frac{2}{a}}\right\}\right)\right\}\right\}$ be a fuzzy soft topology on $X$ and $f_{A}=\left\{\left(e_{1},\left\{a_{\frac{1}{a}}, b_{\frac{1}{a}}\right\}\right)\right\}$. Then $f_{A}$ is a $F S C_{2}$-connected ( respectively, $F S C_{3}$-connected) set. But $f_{A}$ is not a $F S C_{S}$-connected as there exist $h_{A}=\left\{\left(e_{1},\left\{b_{1}\right\}\right)\right\}$ and $s_{A}=\left\{\left(e_{1},\left\{a_{\frac{1}{g}}\right\}\right)\right\}$ fuzzy soft weakly separated sets such that $f_{A}=h_{A} \widetilde{\cup} s_{A}$.

Theorem 4.5. Let $(X, \tau, E)$ be a fuzzy soft topological space, $f_{E} \in \operatorname{FSS}(X)_{E}$. If $f_{E}$ is a $F S C_{3}$-connected set in $X$, then $f_{E}$ is a $F S C_{M}$-connected.

Proof. Let $f_{E}$ be a $F S C_{3}$-connected set in $X$. Suppose $f_{E}$ is a $F S C_{M}$-disconnected. There exist non-null fuzzy soft $Q$-separated sets $h_{E}$ and $s_{E}$ in $X$ such that $f_{E}=h_{E} \widetilde{\cup}_{s_{E}}$. Let $g_{E}=\left[\mathrm{Fcl}\left(h_{E}\right)\right]^{c}$ and $u_{E}=\left[\mathrm{Fcl}\left(s_{E}\right)\right]^{c}$. Then $g_{E}$ and $u_{E}$ are non-null fuzzy soft open sets.

Now, $g_{E} \widetilde{\sim} u_{E}=\left[F \operatorname{cl}\left(h_{E}\right)\right]^{c} \widetilde{\sim}\left[F c l\left(s_{E}\right)\right]^{c}=\left[F \operatorname{cl}\left(h_{E}\right)^{c} \widetilde{\sim}\left[\operatorname{Fcl}\left(s_{E}\right)\right]^{c}\right.$ $=\left[\operatorname{Fcl}\left(h_{E} \widetilde{\cup} s_{E}\right)\right]^{c} \subseteq\left(f_{E}\right)^{c}$.

Also, $g_{E} \not \subset\left(f_{E}\right)^{c}$. For, if $g_{E} \subseteq f_{E}^{c}$, then $f_{E} \subseteq g_{E}^{c}=\operatorname{Fcl}\left(h_{E}\right)$ which whould imply $s_{E}=\tilde{0}_{E}$ (since $\left.\boldsymbol{F} c l\left(h_{E}\right) \widetilde{\cap} s_{E}=\widetilde{\mathrm{O}}_{E}\right)$. This is a contradiction. Similarly, $u_{E} \not \subset\left(f_{E}\right)^{c}$.

Therefore, $f_{E}$ is a $F S C_{3}$-disconnected. So, $f_{E}$ is a $F S C_{M}$-connected. 
Remark 4.4. If $f_{E}$ is a $F S C_{M}$-connected, then it may not be a $F S C_{3}$-connected as shown by the following example.

Example 4.4. Let $X=\{a, b\}, E=\left\{e_{1}, e_{2}\right\}, A=\left\{e_{1}\right\} \subseteq E, \tau=\left\{\tilde{1}_{E}, \tilde{0}_{E},\left\{\left(e_{1},\left\{a_{0.6}, b_{0.2}\right\}\right)\right\},\left\{\left(e_{1},\left\{a_{0.2}\right.\right.\right.\right.$, $\left.\left.\left.\left.b_{0.7}\right\}\right)\right\},\left\{\left(e_{1},\left\{a_{0.6}, b_{0.7}\right\}\right)\right\},\left\{\left(e_{1},\left\{a_{0.2}, b_{0.2}\right\}\right)\right\}\right\}$ be a fuzzy soft topology on $X$ and $f_{A}=\left\{\left(e_{1},\left\{a_{0.5}, b_{0.7}\right\}\right)\right\}$. Then there exist non-null fuzzy soft open sets $h_{A}=\left\{\left(e_{1},\left\{a_{0.2}, b_{0.2}\right\}\right)\right\}$ and $s_{A}=\left\{\left(e_{1},\left\{a_{0.2}, b_{0.7}\right\}\right)\right\}$ such that $f_{A} \subseteq h_{A} \sim_{s_{A}}, h_{A} \tilde{\cap} s_{A} \subseteq\left(f_{A}\right)^{c}, h_{A} \not \subset\left(f_{A}\right)^{c}$ and $s_{A} \not \subset\left(f_{A}\right)^{c}$. So, $f_{A}$ is not a $F S C_{3}$. connected. However, $f_{A}$ is a $F S C_{M}$-connected.

Theorem 4.6. Let $(X, \tau, E)$ be a fuzzy soft topological space. A fuzzy soft set $f_{E}$ in $X$ is a $F S C_{2}$-connected if and only if $f_{E}$ is a $F S O$-connected.

Proof. Let $f_{E}$ be a $F S C_{2}$-connected set in $X$. Suppose $f_{E}$ is not a $F S O$-connected. Then there exist non-null fuzzy soft separated sets $h_{E}$ and $s_{E}$ in $X$ such that $f_{E}=h_{E} \widetilde{\cup} s_{E}$. By Theorem 3.1 and Remark 3.3, there exist two nonnull fuzzy soft open sets $g_{E}$ and $u_{E}$ such that $h_{E} \subseteq g_{E}, s_{E} \subseteq u_{E}$, and $g_{E} \tilde{\cap} s_{E}=u_{E} \tilde{\cap} h_{E}=\widetilde{0}_{E}$. Then, $f_{E} \subseteq g_{E} \widetilde{\sim} u_{E}$.

Now, $f_{E} \tilde{\cap} g_{E} \tilde{\cap} u_{E}=\left(h_{E} \tilde{\cap} s_{E}\right) \tilde{\cap} g_{E} \tilde{\cap} u_{E}=\left(h_{E} \tilde{\cap} g_{E} \tilde{\cap} u_{E}\right) \tilde{\cup}\left(s_{E} \tilde{\cap} g_{E} \tilde{\cap} u_{E}\right) \quad=\quad \tilde{0}_{E} \quad$ and $f_{E} \tilde{\cap} g_{E}=\left(h_{E} \widetilde{\cup} s_{E}\right) \tilde{\cap} g_{E}=\left(h_{E} \tilde{\Omega} g_{E}\right) \widetilde{\cup}\left(s_{E} \tilde{\cap} g_{E}\right)=h_{E} \neq \widetilde{0}_{E}$. Similarly, $f_{E} \tilde{\cap} u_{E} \neq \tilde{0}_{E}$ So, $f_{E}$ is not a $F S C_{2}$-connected which is a contradiction.

Conversely, let $f_{E}$ be a $F S O$-connected. Suppose $f_{E}$ is not a $F S C_{2}$-connected. There exist two non-null fuzzy soft open sets $g_{E}, u_{E}$ such that $f_{E} \subseteq g_{E} \tilde{\cup} u_{E}, f_{E} \tilde{\cap} g_{E} \tilde{\cap} u_{E}=\widetilde{0}_{E}, f_{E} \tilde{\cap} u_{E} \neq \widetilde{0}_{E}$, and $f_{E} \tilde{\cap} g_{E} \neq \tilde{0}_{E}$. Hence, $\quad f_{E}=h_{E} \widetilde{\cup} s_{E} \quad$ where $\quad h_{E}=f_{E} \tilde{\cap} g_{E} \subseteq g_{E}$ and $\quad s_{E}=f_{E} \tilde{\cap} u_{E} \subseteq u_{E}$. Also, $g_{E} \tilde{\cap} s_{E}=g_{E} \tilde{\cap}\left(f_{E} \tilde{\cap} u_{E}\right)=\tilde{0}_{E}$. Similarly, $g_{E} \tilde{\cap} u_{E}=\widetilde{0}_{E}$. So, $f_{E}$ is not a FSO -connected and this complete the proof.

Theorem 4.7. Let $(X, \tau, E)$ be a fuzzy soft topological space, $f_{E} \in F S S(X)_{E}$. If $f_{E}$ is a $F S C_{4}$-connected set in $X$, then $f_{E}$ is a $\mathrm{FSO}_{q}$-connected.

Proof. Let $f_{E}$ be a $F S C_{4}$-connected set in $X$. Suppose $f_{E}$ is a $F S O_{q}$-disconnected. Then there exist non-null fuzzy soft strongly separated sets $h_{E}, s_{E}$ in $X$ such that $f_{E}=h_{E}$ ò $s_{E}$.

So, there exist two non-null fuzzy soft open sets $g_{E}$ and $u_{E}$ such that $h_{E} \subseteq g_{E}, s_{E} \subseteq u_{E}$, $g_{E} \widetilde{\cap} s_{E}=u_{E} \widetilde{\cap} h_{E}=\widetilde{0}_{E}, h_{E}$ and $g_{E}$ quasi-coincident with respect to $h_{E}$, and $s_{E}$ and $u_{E}$ quasi-coincident with respect to $s_{E}$. Then, for every $x \in S\left(h_{E}(e)\right)$ we have $\mu_{h_{E}}^{e}(x)+\mu_{g_{E}}^{e}(x)>1$ and for every $x \in S\left(s_{E}(e)\right)$ we have $\mu_{s_{E}}^{e}(x)+\mu_{u_{E}}^{e}(x)>1$. Then, $f_{E} \subseteq g_{E} \widetilde{\cup} u_{E}$. Also, $f_{E} \tilde{\cap} g_{E} \tilde{\cap} u_{E}=\widetilde{0}_{E}$.

Again, $\mu_{g_{E}}^{e}(x)+\mu_{f_{E}}^{e}(x) \geq \mu_{h_{E}}^{e}(x)+\mu_{g_{E}}^{e}(x)>1$ for every $x \in S\left(h_{E}(e)\right)$. Therefore, $g_{E} \not \subset f_{E}^{e}$. Similarly, $u_{E} \not \subset f_{E}^{c}$. Thus, $f_{E}$ is not a $F S C_{4}$-connected. This is a contradiction. So, $f_{E}$ is a $F S O_{q}$-connected.

Remark 4.5. If $f_{E}$ is a $\mathrm{FSO}_{q}$-connected, then it may not be a $\mathrm{FSC}_{4}$-connected as shown by the following example.

Example 4.5. Let $X=\{a, b, c\}, E=\left\{e_{1}, e_{2}\right\}, A=\left\{e_{1}\right\} \subseteq E$ and $\tau=\left\{\tilde{1}_{E}, \tilde{0}_{E},\left\{\left(e_{1},\left\{a_{0.7}\right\}\right)\right\},\left\{\left(e_{1},\left\{b_{0.7}\right.\right.\right.\right.$, $\left.\left.\left.\left.c_{0.8}\right\}\right)\right\},\left\{\left(e_{1},\left\{a_{0.7}, b_{0.7}, c_{0.8}\right\}\right)\right\}\right\}$ be a fuzzy soft topology on $X$. Let $f_{A}=\left\{\left(e_{1},\left\{a_{0.6}, b_{0.4}, c_{0.3}\right\}\right)\right\}$ and $g_{A}=\left\{\left(e_{1},\left\{a_{0.7}\right\}\right)\right\}, \quad u_{A}=\left\{\left(e_{1}, \quad\left\{b_{0.7}, c_{0.8}\right\}\right)\right\} \in \tau$. Then, $f_{A} \subseteq g_{A} \widetilde{\cup} u_{A}, \quad f_{A} \widetilde{\cap} g_{A} \widetilde{\cap} u_{A}=\widetilde{0}_{E}$, $g_{A} \not \subset f_{A}^{o}$ and $u_{N} \not \subset f_{A}^{o}$. So, $f_{A}$ is not a $F S C_{4}$-connected. However, $f_{A}$ is a $F S O_{q}$-connected.

Remark 4.6. If $f_{A}$ is a $F S C_{M}$-connected, then it may not be a $F S O_{q}$-connected as shown by the following example.

Example 4.6. Let $X=\{a, b, c\}, E=\left\{e_{1}, e_{2}\right\}, A=\left\{e_{1}\right\} \subseteq E$ and $\tau=\left\{\tilde{1}_{E}, \tilde{0}_{E},\left\{\left(e_{1},\left\{a_{0.8}\right\}\right)\right\},\left\{\left(e_{1},\left\{b_{0.9}\right.\right.\right.\right.$, $\left.\left.\left.\left.c_{0.9}\right\}\right)\right\},\left\{\left(e_{1},\left\{a_{0.8}, b_{0.9}, c_{0.9}\right\}\right)\right\}\right\}$ be a fuzzy soft topology on $X$. Let $f_{A}=\left\{\left(e_{1},\left\{a_{0.6}, b_{0.7}, c_{0.8}\right\}\right)\right\}$. Then there 
exist two non-null fuzzy soft strongly separated $h_{A}=\left\{\left(e_{1},\left\{a_{0.6}\right\}\right)\right\}$ and $s_{A}=\left\{\left(e_{1},\left\{b_{0.7}, c_{0.8}\right\}\right)\right\}$ such that $f_{A}=h_{A} \tilde{\cup}_{s_{A}}$. So, $f_{A}$ is not a $F S O_{q}$-connected. However, $f_{A}$ is a $F S C_{M}$-connected as $\operatorname{Fcl}\left(s_{A}\right) \widetilde{\cap} h_{A} \neq \widetilde{\mathrm{O}}_{E}$ and also $\operatorname{Fcl}\left(h_{A}\right) \tilde{\cap} s_{A} \neq \widetilde{\mathrm{O}}_{E}$.

Remark 4.7. If $f_{A}$ is a $F S C_{2}$-connected, then it may not be a $F S C_{M}$-connected as shown by the following example.

Example 4.7. Let $X=\{a, b\}, E=\left\{e_{1}\right\}$ and $\tau=\left\{\tilde{1}_{E}, \tilde{0}_{E},\left\{\left(e_{1},\left\{a_{\frac{1}{a}}, b_{1}\right\}\right)\right\},\left\{\left(e_{1},\left\{a_{1}, b_{\frac{1}{g}}\right\}\right)\right\},\left\{\left(e_{1}\right.\right.\right.$, $\left.\left.\left.\left\{a_{1}, b_{\frac{1}{a}}\right\}\right)\right\}\right\}$ be a fuzzy soft topology on $X$. Let $f_{E}=\left\{\left(e_{1},\left\{a_{a}, b_{\frac{2}{g}}\right\}\right)\right\}$. Then $f_{E}$ can be expresed as a union of two

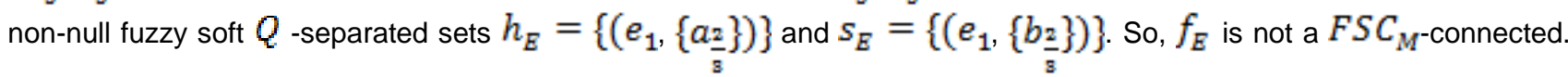
However, $f_{E}$ is a $F S C_{2}$-connected as if we take $g_{E}=\left\{\left(e_{1},\left\{a_{\frac{1}{3}}, b_{1}\right\}\right)\right\}$ and $u_{E}=\left\{\left(e_{1},\left\{a_{1}, b_{\frac{1}{g}}\right\}\right)\right\} \in \tau$, then $f_{E} \subseteq g_{E} \widetilde{\cup} u_{E}$ but $f_{E} \tilde{\cap} g_{E} \widetilde{\cap} u_{E} \neq \widetilde{\mathrm{O}}_{E}$.

Remark 4.8. If $f_{A}$ is a $F S O_{q}$-connected, then it may not be a $F S C_{M}$-connected as shown by the following example.

Example 4.8. Let $X=\{a, b\}, E=\left\{e_{1}, e_{2}\right\}, A=\left\{e_{1}\right\} \subseteq E$ and $\tau=\left\{\tilde{1}_{E}, \tilde{0}_{E},\left\{\left(e_{1},\left\{a_{0,4}\right\}\right)\right\}\right.$, $\left\{\left(e_{1}\right.\right.$, $\left.\left.\left.\left\{b_{0.4}\right\}\right)\right\},\left\{\left(e_{1},\left\{a_{0.4}, b_{1}\right\}\right)\right\},\left\{\left(e_{1},\left\{a_{1}, b_{0.4}\right\}\right)\right\},\left\{\left(e_{1},\left\{a_{0.4}, b_{0.4}\right\}\right)\right\},\left\{\left(e_{1},\left\{a_{1}, b_{1}\right\}\right)\right\}\right\}$ be a fuzzy soft topology on $X$. Let $f_{A}=\left\{\left(e_{1},\left\{a_{0.4}, b_{0.4}\right\}\right)\right\}$. Then there exist two non-null fuzzy soft $Q$-separated sets $h_{A}=\left\{\left(e_{1},\left\{a_{0.4}\right\}\right)\right\}$ and

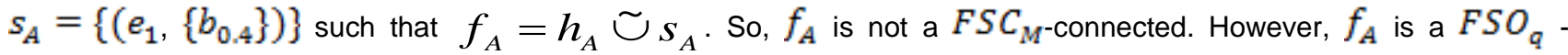
connected as $h_{A}$ and $s_{A}$ are not fuzzy soft strongly separated.

Remark 4.9. If $f_{A}$ is a $F S C_{5}$-connected set, then it may not be a $F S O$-connected (respectively, $F S O_{q}$-connected, $F S C_{i}$-connected for $i=1,2,3,4, S, M$ ) set. In fact, $f_{A}$ defined in Example 4.6 (or 4.7) is a $F S C_{5}$-connected, but it is a not a $\mathrm{FSO}_{\mathrm{q}}$-connected set and not a SSC $_{M^{-}}$-connected set. Therefore, it is not a $F S O$-connected set and not a FSC $_{i^{-}}$ connected set for $i=1,2,3,4, S$.

Remark 4.10. If $f_{A}$ is a $F S O$-connected (respectively, $F S O_{q}$-connected, $F S C_{\tilde{i}}$-connected for $i=1,2,3,4, S, M$ ) set, it may not be a a $F_{5} C_{5}$-connected as shown by the following example.

Example 4.9. Let $X=\{a, b\}, E=\left\{e_{1}, e_{2}\right\}, A=\left\{e_{1}\right\} \subseteq E$ and $\tau=\left\{\tilde{1}_{E}, \tilde{0}_{E},\left\{\left(e_{1},\left\{a_{0,3}\right\}\right)\right\}\right.$, $\left\{\left(e_{1}\right.\right.$, $\left.\left.\left.\left\{a_{0.6}, b_{0.5}\right\}\right)\right\}\right\}$ be a fuzzy soft topology on $X$. Let $f_{A}=\left\{\left(e_{1},\left\{b_{0.7}\right\}\right)\right\}$. Then $f_{A}$ is a $F S C_{i}$-connected for $i=1,2,3,4, S, M$. But since $\left\{\left(e_{1},\left\{b_{0.5}\right\}\right)\right\}$ is a non-null proper clopen fuzzy soft set in $f_{A}$. So, $f_{A}$ is not a $F S C_{5-}$ connected.

Remark 4.11. In a fuzzy soft topological space $(X, \tau, E)$. The classes of $F S O$-connected, $F S O_{q}$-connected, and $F S C_{i}$-connected sets for $i=1,2,3,4, S, M$ can be discribed by the following diagram.

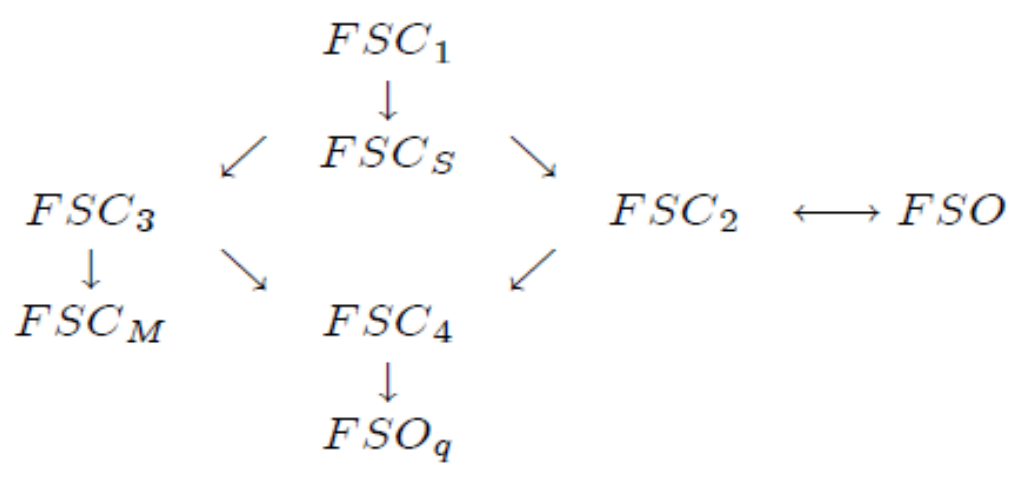

We observe that, if a fuzzy soft point $x_{\alpha}^{e}$ is $F S C_{i}$-connected set $(i=2,3)$ hence $F S C_{4}$-connected, but not necessarily $F S C_{1}$-connected which is a departure from general topology where points are connected sets. 
Example 4.10. Let $X=\left\{a_{,} b\right\}, E=\left\{e_{1}, e_{2}\right\}, \tau=\left\{\tilde{1}_{E}, \tilde{0}_{E},\left\{\left(e_{1},\left\{a_{\frac{1}{a}}, b_{\frac{z}{a}}\right\}\right)\right\},\left\{\left(e_{1},\left\{a_{\frac{x}{a}}, b_{\frac{1}{a}}\right\}\right)\right\},\left\{\left(e_{1},\left\{a_{\frac{x}{a}}\right.\right.\right.\right.$, $\left.\left.\left.\left.b_{\frac{2}{a}}\right\}\right)\right\},\left\{\left(e_{1},\left\{a_{\frac{1}{a}}, b_{\frac{1}{a}}\right\}\right)\right\}\right\}$. Here, the fuzzy soft point $\frac{a_{\frac{1}{g}}^{e_{1}}}{g}=\left\{\left(e_{1},\left\{\frac{a_{1}}{g}\right\}\right)\right\}$ is not a $F S C_{1}$-connected

Moreover, we observe that the null-fuzzy soft set $\tilde{0}_{E}$ is $F S C_{1}$-connected and hence $F S C_{i}$-connected $(i=2,3,4)$.

Theorem 4.8. Let $(X, \tau, E)$ and $(Y, \sigma, K)$ be a fuzzy soft topological spaces and $f_{p u}:(X, \tau, E) \rightarrow(Y, \sigma, K)$ be fuzzy soft bijective continuos mapping. If $g_{B}$ is a $F S C_{i}$-connected (respectively, $F S O$-connected, $F S O_{q^{-} \text {-connected) set }}$ in $X$ for $i=5, S, M$, then $f_{p u}\left(g_{B}\right)$ is a $F S C_{i}$-connected (respectively, FSO -connected, $F S O_{q}$-connected) set in $Y$ for $i=5, S, M$.

Proof. As a sample, we will prove the case $i=5$. Let $g_{B}$ be a $F S C_{5}$-connected set in $X$. Suppose, $f_{p u}\left(g_{B}\right)$ is not a $F_{S C}$-connected set in $Y$. Then, $f_{p u}\left(g_{B}\right)$ has a non-null proper clopen fuzzy soft subset $h_{C}$.

So, there exist $s_{D} \in \sigma$ and $u_{N} \in \sigma^{\sigma}$ such that $h_{C}=f_{p u}\left(g_{B}\right) \widetilde{\cap} s_{D}=f_{p u}\left(g_{B}\right) \widetilde{\cap} u_{N}$. Since $f_{p u}$ is a bijective function, then $f_{p u}^{-1}\left(h_{C}\right)=g_{B} \tilde{\cap} f_{p u}^{-1}\left(s_{D}\right)=g_{B} \tilde{\cap} f_{p u}^{-1}\left(u_{N}\right)$.

Also, since $s_{D} \in \sigma$ and $u_{N} \in \sigma^{\sigma}$ and $f_{p u}$ is a fuzzy soft continuos function, then $f_{p u}^{-1}\left(s_{D}\right) \in \tau$ and $f_{p u}^{-1}\left(u_{N}\right) \in \tau^{\sigma}$. Hence, $f_{p u}^{-1}\left(h_{C}\right)$ is a non-null proper clopen fuzzy soft subset of $g_{B}$ which is a contradiction. Therefore, $f_{p u}\left(g_{B}\right)$ is a FSC - $_{5}$-connected set in $Y$.

Theorem 4.9. Let $(X, \tau, E)$ and $(Y, \sigma, K)$ be a fuzzy soft topological spaces and $f_{p u}:(X, \tau, E) \rightarrow(Y, \sigma, K)$ be fuzzy soft open function such that $p: E \rightarrow K, u: X \rightarrow Y$ are bijetive mapping. If $g_{B}$ is a $F S C_{i}$-connected (respectively, FSO -connected, $F S O_{q}$-connected) set in $Y$ for $i=1,2,3,4,5, M, S$, then $f_{p u}^{-1}\left(g_{B}\right)$ is a FSC $C_{i}$-connected

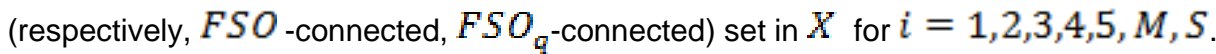

Proof. As a sample, we will prove the case of FSO -connected. Let $g_{B}$ be a FSO -connected set in $Y$. Suppose $f_{p u}^{-1}\left(g_{B}\right)$ is not a FSO -connected set in $X$. Then there exist two non-null fuzzy soft separated sets $h_{C}$ and $s_{D}$ in $X$ such that $f_{p u}^{-1}\left(g_{B}\right)=h_{C} \widetilde{\cup}_{s_{D}}$. Therefore, there exist two non-null fuzzy soft open sets $u_{N}$ and $j_{L}$ in $X$ such that $h_{C} \subseteq u_{N}, s_{D} \subseteq j_{L}$ and $h_{C} \tilde{\cap} j_{L}=s_{D} \tilde{\cap} u_{N}=\widetilde{0}_{E}$. Since $f_{p u}$ is a surjective fuzzy soft function, then $f_{p u}\left[f_{p u}^{-1}\left(g_{B}\right)\right]=g_{B}$ and so $g_{B}=f_{p u}\left[h_{C} \tilde{\cup}_{s_{D}}\right]=f_{p u}\left(h_{C}\right) \widetilde{\cup} f_{p u}\left(s_{D}\right)$. Since $f_{p u}$ is a fuzzy soft open function, then $f_{p u}\left(u_{N}\right)$ and $f_{p u}\left(j_{L}\right)$ are non-null fuzzy soft open sets in $Y$ such that $f_{p u}\left(h_{C}\right) \subseteq f_{p u}\left(u_{N}\right)$, $f_{p u}\left(s_{D}\right) \subseteq f_{p u}\left(j_{L}\right)$. Since $f_{p u}$ is a fuzzy soft injective function, then $f_{p u}\left(h_{C}\right) \tilde{\cap} f_{p u}\left(j_{L}\right)=f_{p u}\left(h_{C} \tilde{\cap} j_{L}\right)=\widetilde{0}_{K}$ and $f_{p u}\left(s_{D}\right) \tilde{\cap} f_{p u}\left(s_{D}\right)=\widetilde{0}_{K}$. It follows that $g_{B}$ is a FSO-disconnected set, which a contradiction.

Definition 4.6. Two non-null fuzzy soft sets $f_{A}$ and $g_{B}$ are said to be intersecting if there exist $x \in X, e \in E$ such that $\min \left\{f_{A}(x)(e), g_{B}(x)(e)\right\} \neq 0$. If $f_{A}$ and $g_{B}$ are non-intersecting, then $f_{A}$ and $g_{B}$ are said to be disjoint.

Theorem 4.10. If $f_{A}$ and $g_{B}$ are intersecting $F S C_{1}$-connected (respectively, $F S C_{2}$-connected, $F S O$-connected, $F S C_{S}$-connected, $F S C_{M}$-connected, $F S O_{q}$-connected) sets in $X$, then $f_{A} \widetilde{\cup} g_{B}$ is a $F S C_{1}$-connected

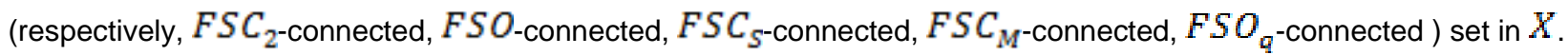

Proof. The cases of $F S C_{1}$-connected and $F S C_{2}$-connected sets previously proved (see Theorem 3.22, 3.23 and 3.24 in [9] ). As a sample we will prove the case of $F S C_{M^{-}}$connected sets. Let $f_{A}$ and $g_{B}$ be intersecting $F S C_{M}$-connected sets in $X$. Suppose $f_{A} \widetilde{\cup} g_{B}$ is a $F S C_{M}$-disconnected set. Then, there exist two non-null fuzzy soft $Q$-separated sets $h_{C}$ and $s_{D}$ in $X$ such that $f_{A} \widetilde{\cup} g_{B}=h_{C} \widetilde{\cup} s_{D}$. Therefore, $f_{A} \tilde{\cap} h_{C}, f_{A} \tilde{\cap} s_{D}, g_{B} \tilde{\cap} h_{C}$ and $g_{B} \tilde{\cap} s_{D}$ are non-null fuzzy soft $Q$-separated sets in $X$ as subsets of $h_{C}$ and $s_{D}$. Since $f_{A}=\left(f_{A} \tilde{\cap} h_{C}\right) \widetilde{\cup}\left(f_{A} \tilde{\curvearrowright} s_{D}\right)$ and $g_{B}=\left(g_{B} \tilde{\cap} h_{C}\right) \widetilde{\cup}\left(g_{B} \tilde{\cap} s_{D}\right)$, then $f_{A}$ and $g_{B}$ are $F S C_{M}$ disconnected which is a contradiction. 
Theorem 4.11. Let $\left\{\left(f_{A}\right)_{i} ; i \in J\right\}$ be a family of a $F S C_{1}$-connected (respectively, $F S C_{2}$-connected, FSO . connected, $F S C_{S}$-connected, $F S C_{M}$-connected, $F S O_{q}$-connected ) sets in $X$ such that for $i, j \in J ; i \neq j$ the fuzzy soft sets $\left(f_{A}\right)_{i}$ and $\left(f_{A}\right)_{j}$ are intersecting. Then, $f_{A}=\cup_{i \in J}\left(f_{A}\right)_{i}$ is a $F S C_{1}$-connected (respectively, $F S C_{2}$ connected, $F S O$-connected, SSC $_{S}$-connected, $F_{\text {S }}$-connected, FSO $_{\mathrm{q}}$-connected) set in $X$.

Proof. Let $\left\{\left(f_{A}\right)_{i} ; i \in J\right\}$ be a family of a $F S C_{1}$-connected sets in $X$. Suppose that $f_{A}$ is not a $F S C_{1}$-connected set in $X$. Then, there exist two fuzzy soft open sets $h_{C}$ and $S_{D}$ in $X$ such that $f_{A} \subseteq h_{C} \widetilde{\cup}_{S_{D}}$ and $h_{C} \tilde{\cap} s_{D} \subseteq f_{A}{ }^{c}$

Now, let $\left(f_{A}\right)_{i_{z}}$ be any fuzzy soft set of the given family. Then, $\left(f_{A}\right)_{i_{0}} \subseteq h_{C} \widetilde{\cup} s_{D}$ and $h_{C} \widetilde{\cap} s_{D} \subseteq\left(f_{A}\right)_{i_{0}}^{c}$. But,

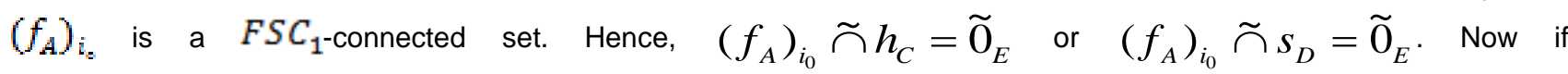
$\left(f_{A}\right)_{i_{0}} \tilde{\cap} h_{C}=\widetilde{0}_{E}$, we can prove that $\left(f_{A}\right)_{i} \tilde{\cap} h_{C}=\widetilde{0}_{E}$ for each $i \in J-\left\{i_{\odot}\right\}$ and so $f_{A} \tilde{\cap} h_{C}=\widetilde{0}_{E}$. This complete the proof.

Corollary 4.1. If $\left\{\left(f_{A}\right)_{i} ; i \in J\right\}$ is a family of a $F S C_{1}$-connected (respectively, $F S C_{2}$-connected, $F S O$-connected, FSC $C_{S}$-connected, $F S C_{M}$-connected, $F S O_{q}$-connected) in $X$ and $\underset{i \in J}{\cap}\left(f_{A}\right)_{i} \neq \widetilde{\mathrm{O}}_{E}$, then $\underset{i \in J}{\cup}\left(f_{A}\right)_{i}$ is a $F S C_{1^{-}}$ connected (respectively, $F_{2}$-connected, $F S O$-connected, $F_{S} C_{S}$-connected, $F_{\text {SC }}$-connected, FSO $_{q}$-connected) set in $X$.

Proof. Straightforward in view of Theorem 4.10.The following example shows that Theorem 4.10 fails for $F_{3}$. connected (respectively, $\mathrm{FSC}_{4}$-connected) spaces.

Example 4.11. Let $X=\{a, b\}, E=\left\{e_{1}, e_{2}\right\}, A=\left\{e_{1}\right\} \subseteq E$ and $\tau=\left\{\tilde{1}_{E}, \tilde{0}_{E},\left\{\left(e_{1},\left\{a_{\frac{4}{5}}, b_{\frac{z}{5}}\right\}\right)\right\},\left\{\left(e_{1},\left\{a_{\frac{z}{5}}\right.\right.\right.\right.$, $\left.\left.\left.\left.b_{\frac{4}{5}}\right\}\right)\right\},\left\{\left(e_{1},\left\{a_{\frac{2}{5}}, b_{\frac{2}{5}}\right\}\right)\right\},\left\{\left(e_{1},\left\{a_{\frac{4}{5}}, b_{\frac{4}{5}}\right\}\right)\right\}\right\}$ be a fuzzy soft topology on $X$. Let $f_{A}=\left\{\left(e_{1},\left\{a_{\frac{1}{5}}, b_{\frac{2}{5}}\right\}\right)\right\}$ and $g_{A}$ $=\left\{\left(e_{1},\left\{a_{\frac{\pi}{5}}, b_{\frac{1}{5}}\right\}\right)\right\}$. Here, $f_{A} \widetilde{\cap} g_{A} \neq \widetilde{\mathrm{O}}_{E}$ and $f_{A}$ and $g_{A}$ are $F S C_{3}$-connected sets in $X$, but $f_{A} \widetilde{\cup} g_{A}$ is not $F S C_{3}$-connected set in $X$.

Example 4.12. Let $X=\{a, b\}, E=\left\{e_{1}, e_{2}\right\}$ and $\tau=\left\{\tilde{1}_{E}, \tilde{0}_{E},\left\{\left(e_{1},\left\{\frac{a s}{5}, b_{\frac{2}{5}}\right\}\right)\right\},\left\{\left(e_{2},\left\{\frac{a}{5}, b_{\frac{s}{5}}\right\}\right)\right\},\left\{\left(e_{1},\left\{a_{\frac{s}{5}}\right.\right.\right.\right.$, $\left.\left.\left.\left.b_{\frac{2}{5}}\right\}\right),\left(e_{2},\left\{a_{\frac{2}{5}}, b_{\frac{s}{5}}\right\}\right)\right\}\right\}$ be a fuzzy soft topology on $X$. Let $f_{E}=\left\{\left(e_{1},\left\{a_{\frac{s}{5}}\right\}\right),\left(e_{2},\left\{\frac{a_{2}}{5}\right\}\right)\right\}$ and $g_{E}=\left\{\left(e_{1},\left\{a_{\frac{1}{5}}\right.\right.\right.$, $\left.\left.\left.b_{\frac{2}{5}}^{s}\right\}\right),\left(e_{2},\left\{b_{\frac{s}{5}}^{s}\right\}\right)\right\}$. Here, $f_{E} \widetilde{\sim} g_{E} \neq \widetilde{\mathrm{O}}_{E}$ and $f_{E}$ and $g_{E}$ are $F S C_{4}$-connected sets in $X$, but $f_{E} \widetilde{\cup} g_{E}$ is not FSC $_{4}$-connected set in $X$.

Theorem 4.12. If $f_{A}$ and $g_{B}$ are quasi-coincident $F S C_{3}$-connected (respectively, $F S C_{4}$-connected) sets in $X$, then $f_{A} \widetilde{\cup} g_{B}$ is a $F S C_{3}$-connected (respectively, $F S C_{4}$-connected) set in $X$.

Proof. As a sample, we will prove the case $F S C_{3}$-connected. Let $f_{A}$ and $g_{B}$ be quasi-coincident $F S C_{3}$-connected sets in $X$. Suppose there exist two non-null fuzzy soft open sets $h_{C}$ and $s_{D}$ in $X$ such that

$$
f_{A} \widetilde{\cup} g_{B} \subseteq h_{C} \widetilde{\cup} s_{D} \text { and } h_{C} \tilde{\cap} s_{D} \subseteq\left(f_{A} \widetilde{\cup} g_{B}\right)^{c}
$$

Therefore, $f_{A} \subseteq h_{C} \widetilde{\cup} s_{D}, h_{C} \tilde{\cap} s_{D} \subseteq f_{A}{ }^{c}, g_{B} \subseteq h_{C} \widetilde{\cup} s_{D}$ and $h_{C} \tilde{\cap} s_{D} \subseteq g_{B}{ }^{c}$. Since $f_{A}$ and $g_{B}$ are FSC 3 $_{\text {-connected, then }}\left(h_{C} \subseteq f_{A}^{c}\right.$ or $\left.s_{D} \subseteq f_{A}^{c}\right)$ and $\left(h_{C} \subseteq g_{B}^{c}\right.$ or $\left.s_{D} \subseteq g_{B}^{c}\right)$.

Moreover, since $f_{A}$ and $g_{B}$ are quasi-coincident, there exist $x \in X, e \in E$ such that

$$
\mu_{f_{A}}^{e}(x)>1-\mu_{g_{B}}^{e}(x)
$$

Now, consider the following cases: 
Case I. Suppose $h_{C} \subseteq f_{A}^{c}$. Then by (2) we have,

$$
\mu_{h_{C}}^{e}(x)<\mu_{g_{B}}^{e}(x)
$$

We claim that, $s_{D} \not \subset g_{B}^{c}$. For if not, then

$$
\mu_{s_{D}}^{e}(x) \leq 1-\mu_{g_{B}}^{e}(x)<\mu_{f_{A}}^{e}(x)
$$

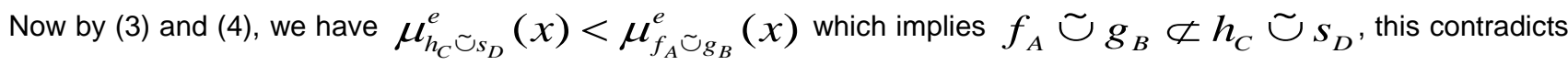
(1). Hence, $h_{C} \subseteq g_{B}^{c}$. Therefore, $h_{C} \subseteq f_{A}{ }^{c} \tilde{\cap} g_{B}{ }^{c}=\left(f_{A} \widetilde{\cup} g_{B}\right)^{c}$.

Case II: Suppose, $s_{D} \subseteq f_{A}^{c}$. Here, we can show as in Case I that $h_{C} \not \subset g_{B}{ }^{c}$. Therefore, $s_{D} \subseteq g_{B}^{c}$. Hence, $s_{D} \subseteq f_{A}{ }^{c} \tilde{\cap} g_{B}{ }^{c}=\left(f_{A} \widetilde{\cup} g_{B}\right)^{c}$. This complete the proof.

Theorem 4.13. Let $\left\{\left(f_{A}\right)_{i} ; i \in J\right\}$ be a family of a $F S C_{3}$-connected (respectively, $F S C_{4}$-connected) sets in $X$ such that for $i_{j} j \in J ; i \neq j$ the fuzzy soft sets $\left(f_{A}\right)_{i}$ and $\left(f_{A}\right)_{j}$ are quasi-coincident. Then $f_{A}=\cup_{i \in J}\left(f_{A}\right)_{i}$ is a $F S C_{3^{-}}$ connected (respectively, $\mathrm{FSC}_{4}$-connected) set in $\mathrm{X}$.

Proof. Let $\left\{\left(f_{A}\right)_{i} ; i \in J\right\}$ be a family of a $F S C_{3}$-connected sets in $X$. Suppose there exist two fuzzy soft open sets $h_{C}$ and $s_{D}$ in $X$ such that $f_{A} \subseteq h_{C} \widetilde{\cup} s_{D}$ and $h_{C} \widetilde{\cap} s_{D} \subseteq f_{A}{ }^{c}$. Let $\left(f_{A}\right)_{i_{z}}$ be any fuzzy soft set of the given family. Then, $\left(f_{A}\right)_{i_{0}} \subseteq h_{C} \widetilde{\cup} s_{D}$ and $h_{C} \tilde{\cap} s_{D} \subseteq\left(f_{A}\right)_{i_{0}}^{c}$. Since $\left(f_{A}\right)_{i_{\varepsilon}}$ is a $F S C_{3}$-connected set, we have $h_{C} \subseteq\left(f_{A}\right)_{i_{0}}^{c}$ or $s_{D} \subseteq\left(f_{A}\right)_{i_{0}}^{c}$. Now, the result follows in view of the facts that $\left(f_{A}\right)_{i_{s}} \subseteq\left(h_{C}\right)^{c}$ then $\left(f_{A}\right)_{i} \subseteq\left(h_{C}\right)^{c}$ for each $i \in J-\left\{i_{\odot}\right\}$, since $\left(f_{A}\right)_{i_{s}}$ and $\left(f_{A}\right)_{i}$ are are quasi-coincident $F S C_{3}$-connected sets, and $h_{C} \subseteq\left[\tilde{\Omega}_{i \in J}\left(f_{A}\right)_{i_{0}}\right]^{c}=f_{A}^{c}$. Hence, $f_{A}$ is a $F S C_{3^{3}}$-connected. Similarly, if $\left\{\left(f_{A}\right)_{i} ; i \in J\right\}$ is a family of a $F S C_{4^{-}}$ connected sets in $X$ such that for $i_{y} j \in J ; i \neq j$ the fuzzy soft sets $\left(f_{A}\right)_{i}$ and $\left(f_{A}\right)_{j}$ are quasi-coincident, then $f_{A}=\cup_{i \in J}\left(f_{A}\right)_{i} \quad$ is a $F S C_{4}$-connected set in $X$. This complete the proof.

Corollary 4.2. Let $\left\{\left(f_{A}\right)_{i} ; i \in J\right\}$ be a family of a $F S C_{3}$-connected (respectively, $F S C_{4}$-connected) sets in $X$ and

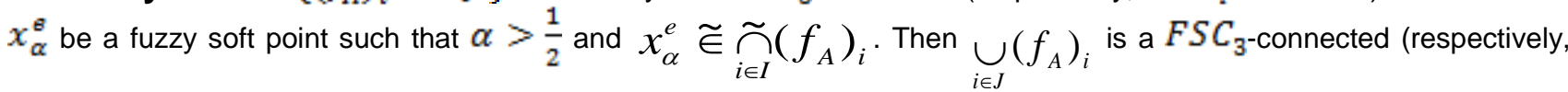
FSC $_{4}$-connected) set in $X$.

Proof. Since $x_{\alpha}^{e} \widetilde{\in} \underset{i \in I}{\widetilde{C}}\left(f_{A}\right)_{i}$, then $x_{\alpha}^{e} \widetilde{\in}\left(f_{A}\right)_{i}$ for each $i \in J$. Therefore, $\left(f_{A}\right)_{i}$ and $\left(f_{A}\right)_{j}$ are quasi-coincident fuzzy soft sets for each $i_{j} j \in J$. By Theorem 4.13, $\cup_{i \in J}\left(f_{A}\right)_{i}$ is a $F S C_{3}$-connected (respectively, $F S C_{4}$-connected) set in $X$.

Theorem 4.15. If $f_{A}$ is a $F S C_{3}$-connected (respectively, $F S C_{4}$-connected, $F S O_{q}$-connected) set in $X$ and $f_{A} \subseteq g_{B} \subseteq F c l\left(f_{A}\right)$, then $g_{B}$ is also a $F S C_{3}$-connected (respectively, $F S C_{4}$-connected, $F S O_{q}$-connected) set in $X$.

Proof. As a sample, we will prove the case of $F S C_{3}$-connected set. Let $h_{C}$ and $s_{D}$ be fuzzy soft open sets in $X$ such that $g_{B} \subseteq h_{C} \widetilde{\cup} s_{D}, h_{C} \tilde{\cap} s_{D} \subseteq g_{B}^{c}$. Then, $f_{A} \subseteq h_{C} \widetilde{\cup} s_{D}$ and $h_{C} \tilde{\cap} s_{D} \subseteq f_{A}^{c}$. Since $f_{A}$ is a

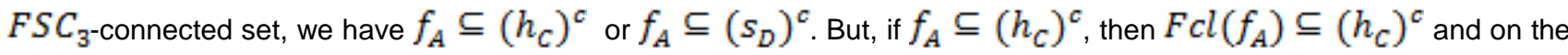
other hand, if $f_{A} \subseteq\left(s_{D}\right)^{c}$, then $\operatorname{Fcl}\left(f_{A}\right) \subseteq\left(s_{D}\right)^{c}$. Therefore, $g_{B} \subseteq \operatorname{Fcl}\left(f_{A}\right) \subseteq\left(h_{C}\right)^{c}$ or $g_{B} \subseteq \operatorname{Fcl}\left(f_{A}\right) \subseteq\left(s_{D}\right)^{c}$. Hence, $g_{B}$ is a FSC $C_{3}$-connected set in $X$. 
However, the above theorem fails in case of $F S C_{1}$-connectedness (respectively, $F S C_{2}$-connectedness, $F S C_{5}$ connectedness, $F S C_{S}$-connectedness, $F S O$-connectedness) which is a departure from general topology. The following example will illustrate that the closure of a $F S C_{1}$-connected (respectively, $F S C_{2}$-connected, $F S C_{5}$-connected, $F S C_{S^{-}}$ connected, $F S O$-connected) set need not be a $F S C_{1}$-connected (respectively, $F S C_{2}$-connected, $F S C_{5}$-connected, $F_{S C}$-connected, FSO -connected). By the following examples we show that Theorem 4.9 and Remark 4.7 of [11] are incorrect.

Example 4.13. Let $X=\{a, b\}, E=\left\{e_{1}\right\}$, and $\tau=\left\{\tilde{1}_{E}, \tilde{0}_{E},\left\{\left(e_{1},\left\{a_{1}\right\}\right)\right\},\left\{\left(e_{1},\left\{b_{z}\right\}\right)\right\},\left\{\left(e_{1},\left\{a_{1}, b_{z}\right\}\right)\right\}\right\}$ be a fuzzy soft topology on $X$. Here, $f_{E}=\left\{\left(e_{1},\left\{a_{1}\right\}\right)\right\}$ is a $F S C_{1}$-connected (respectively, $F S C_{2}$-connected, $F S C_{5}$ connected, $F S C_{S}$-connected, FSO-connected) set, but $\operatorname{Fcl}\left(f_{E}\right)=\left\{\left(e_{1},\left\{a_{1}, b_{\frac{1}{g}}\right\}\right)\right\}$ is not a $F S C_{1}$-connected (respectively, $F S C_{2}$-connected, $F S C_{5}$-connected, $F S C_{S}$-connected, $F S O$-connected).

\section{REFERENCES}

1. Ahmad and A. Kharal, Mappings of Fuzzy Soft Classes, Adv. Fuzzy Syst, (2009).

2. N. Ajmal and J.K. Kohli, Connectedness in fuzzy topological spaces, Fuzzy Sets and Systems 31 (1989) $369-388$.

3. H. Aktaş and N. Çağ̈man, Soft Sets and Soft Groups, Inform. Sci. 177 (2007) 2726-2735.

4. S. Bayramov, C. Gunduz and A.Erdem, Soft Path Connectedness on Soft Topological Spaces, Proceedings of the 13th International Conference on Computational and Mathematical Methods in Science and Engineering, CMMSE 2013.

5. C. L. Chang, Fuzzy Topological Spaces, J. Math. Appl. 24 (1968) 182-193.

6. A.K. Chaudhuri and P. Das, Fuzzy connected sets in fuzzy topological spaces, Fuzzy Sets and Systems 49 (1992) $223-$ 229.

7. U.V. Fatteh and D.S. Bassan, Fuzzy connectedness and its stronger forms, J. Math. Anal. Appl. III (1985) 449-464.

8. S. Hussain, A note on soft connectedness, Journal of Egyption Mathematical Society, 23 (1) 2015, 6-11.

9. S. Karataş, B. Kihç and M. Tellioğlu, On fuzzy soft connected topological spaces, Journal of Linear and Topological Algebra, 3(4) (2015), 229-240.

10. R. Lowen, Fuzzy topological spaces and fuzzy compactness, J. Math. Anal. Appl. 56 (1976) 621-633.

11. J. Mahanta and P. K. Das, Results on Fuzzy Soft Topological Spaces, arXiv:1203.0634v1, 2012.

12. P. K. Maji, R. Biswas, and A.R. Roy, A.R. Fuzzy Soft Sets, J. Fuzzy Math. 9 (3) (2001) 589-602.

13. P. K. Maji, R. Biswas, and A.R. Roy, Soft Set Theory, Computers Math. Appl. 45 (2003) 555-562.

14. P.P. Ming and L.Y. Ming, Fuzzy topology I, Neighbourhood structure of a fuzzy point and Moore-Smith convergence, J. Math. Anal. Appl. 76 (1980) 571-599.

15. D. Molodtsov, Soft Set Theory-First Results, Computers Math. Appl. 37 (4-5) (1999) 19-31.

16. Munir Abdul Khalik Al-Khafaj and Majd Hamid Mahmood, Some Properties of Soft Connected Spaces and Soft Locally Connected Spaces, IOSR Journal of Mathematics, 10 (2014), 102-107.

17. B. Pazar Varol and H. Aygün, Fuzzy Sot Topology, Hacettepe Journal of Mathematics and Statistics, 41 (3) (2012) 407-419.

18. E. Peyghan, B. Samadi and A. Tayebi, On Soft Connectedness, arXiv:1202.1668v1, 2012.

19. S. Roy and T. K. Samanta, An Introduction to Open and Closed Sets on Fuzzy Topological Spaces, Annals of Fuzzy Mathematics and Informatics, 2012.

20. S. Saha, Local connectedness in fuzzy setting, Simon Stevin 61 (1987) 3-13.

21. M. Shabir and M. Naz, On Soft Topological Spaces, Computers and Mathematics with Applications 61 (2011) 17861799.

22. B. Tanay and M. B. Kandemir, Topological Structures of Fuzzy Soft Sets, Computers and Mathematics with Applications 61 ( 2011 ) 412-418.

23. Tridiv Jyotineog and Dusmanta Kumar Sut, Some New Operations of Fuzzy Soft Sets, J. Math. Comput. Sci. 2 (5) 
(2012) 1186-1199.

24. Tugbahan Simsekler, Saziye Yuksel, Fuzzy Soft Topological spaces, Ann. Fuzzy Math. Inform, vol. x(2012) 1-x.

25. Ş. Yüksel, Z. G. Ergül and Z. Güven, Soft Connected Spaces, International J. of Pure \& Eng. Mathematics (IJPEM), 2 (2014), 121-134.

26. L.A. Zadeh, Fuzzy sets, Inform. and Control 8 (1965) 338-353.

27. X.A. Zhao, Connectedness on fuzzy topological spaces, Fuzzy Sets and Systems 20 (1986) 223-240.

28. Zheng Chong You, On connectedness of fuzzy topological spaces, Fuzzy Mathematics 3 (1982) 59-66. 